\title{
Global Well-Posedness of Classical Solutions with Large Oscillations and Vacuum to the Three-Dimensional Isentropic Compressible Navier-Stokes Equations*
}

\author{
Xiangdi HUANG ${ }^{a, c}$, Jing $\mathrm{LI}^{b, c}$, Zhouping $\mathrm{XIN}^{c}$ \\ ${ }^{a}$ Department of Mathematics, \\ University of Science and Technology of China, Hefei 230026, P. R. China \\ ${ }^{b}$ Institute of Applied Mathematics, AMSS, \\ Academia Sinica, Beijing 100190, P. R. China \\ ${ }^{c}$ The Institute of Mathematical Sciences, \\ The Chinese University of Hong Kong, Shatin, Hong Kong
}

\begin{abstract}
We establish the global existence and uniqueness of classical solutions to the Cauchy problem for the isentropic compressible Navier-Stokes equations in three spatial dimensions with smooth initial data which are of small energy but possibly large oscillations with constant state as far field which could be either vacuum or non-vacuum. The initial density is allowed to vanish and the spatial measure of the set of vacuum can be arbitrarily large, in particular, the initial density can even have compact support. These results generalize previous results on classical solutions for initial densities being strictly away from vacuum, and are the first for global classical solutions which may have large oscillations and can contain vacuum states.
\end{abstract}

\section{Introduction}

The time evolution of the density and the velocity of a general viscous isentropic compressible fluid occupying a domain $\Omega \subset \mathbb{R}^{3}$ is governed by the compressible NavierStokes equations:

$$
\left\{\begin{array}{l}
\rho_{t}+\operatorname{div}(\rho u)=0 \\
(\rho u)_{t}+\operatorname{div}(\rho u \otimes u)-\mu \Delta u-(\mu+\lambda) \nabla(\operatorname{div} u)+\nabla P(\rho)=0,
\end{array}\right.
$$

where $\rho \geq 0, u=\left(u^{1}, u^{2}, u^{3}\right)$ and $P=a \rho^{\gamma}(a>0, \gamma>1)$ are the fluid density, velocity and pressure, respectively. The constant viscosity coefficients $\mu$ and $\lambda$ satisfy the physical restrictions:

$$
\mu>0, \quad \mu+\frac{3}{2} \lambda \geq 0
$$

${ }^{*}$ This research is supported in part by Zheng Ge Ru Foundation, Hong Kong RGC Earmarked Research Grants CUHK4040/06P and CUHK4042/08P, and a Focus Area Grant from The Chinese University of Hong Kong. The research of J. Li is partially supported by NSFC Grant No. 10971215. Email: xdhuang@ustc.edu.cn (X. Huang), ajingli@gmail.com (J. Li), zpxin@ims.cuhk.edu.hk (Z. Xin). 
Let $\Omega=\mathbb{R}^{3}$ and $\tilde{\rho}$ be a fixed nonnegative constant. We look for the solutions, $(\rho(x, t), u(x, t))$, to the Cauchy problem for (1.1) with the far field behavior:

$$
u(x, t) \rightarrow 0, \quad \rho(x, t) \rightarrow \tilde{\rho} \geq 0, \quad \text { as }|x| \rightarrow \infty
$$

and initial data,

$$
\left.(\rho, u)\right|_{t=0}=\left(\rho_{0}, u_{0}\right), \quad x \in \mathbb{R}^{3} .
$$

There are huge literatures on the large time existence and behavior of solutions to (1.1). The one-dimensional problem has been studied extensively by many people, see $[9,21,31,32$ and the references therein. For the multi-dimensional case, the local existence and uniqueness of classical solutions are known in [28, 33] in the absence of vacuum and recently, for strong solutions also, in [3, 5, 6, 30] for the case that the initial density need not be positive and may vanish in open sets. The global classical solutions were first obtained by Matsumura-Nishida [27] for initial data close to a nonvacuum equilibrium in some Sobolev space $H^{s}$. In particular, the theory requires that the solution has small oscillations from a uniform non-vacuum state so that the density is strictly away from the vacuum and the gradient of the density remains bounded uniformly in time. Later, Hoff [10,11] studied the problem for discontinuous initial data. For the existence of solutions for arbitrary data (the far field density is vacuum, that is, $\tilde{\rho}=0$ ), the major breakthrough is due to Lions [26] (see also Feireisl [7]), where he obtains global existence of weak solutions - defined as solutions with finite energy when the exponent $\gamma$ is suitably large. The main restriction on initial data is that the initial energy is finite, so that the density vanishes at far fields, or even has compact support. However, little is known on the structure of such weak solutions. Recently, under the additional assumptions that the viscosity coefficients $\mu$ and $\lambda$ satisfy

$$
\mu>\max \{4 \lambda,-\lambda\}
$$

and for the far field density away from vacuum $(\tilde{\rho}>0)$, Hoff $([12,14,15])$ obtained a new type of global weak solutions with small energy, which have extra regularity information compared with those large weak ones constructed by Lions ( [26]) and Feireisl ( [7]). Note that here the weak solutions may contain vacuum though the spatial measure of the set of vacuum has to be small. Moreover, under some additional conditions which prevent the appearance of vacuum states in the data, Hoff ( [12,15]) obtained also classical solutions.

It should be noted that in the presence of vacuum, the global well-posedness of classical solutions and the regularity and uniqueness of those weak solutions ( $7,12,26])$ remains completely open. Indeed, this is a subtle issue since, in general, one would not expect such general results due to Xin's blow-up results in [34, where it is shown that in the case that the initial density has compact support, any smooth solution to the Cauchy problem of the non-barotropic compressible Navier-Stokes system without heat conduction blows up in finite time for any space dimension, and the same holds for the isentropic case (1.1), at least in one-dimension, and the symmetric two-dimensional case ( [18]). See also the recent generalizations to the cases for the non-barotropic compressible Navier-Stokes system with heat conduction ( [4]) and for non-compact but rapidly decreasing at far field initial densities ( [29]).

In this paper, we will study the global existence and uniqueness of classical solutions to the Cauchy problem for the isentropic compressible Navier-Stokes equations, (1.1), in three-dimensional space with smooth initial data which are of small energy but possibly 
large oscillations with constant state as far field which could be either vacuum $(\tilde{\rho}=0)$ or non-vacuum $(\tilde{\rho}>0)$; in particular, the initial density is allowed to vanish, even has compact support.

Before stating the main results, we explain the notations and conventions used throughout this paper. We denote

$$
\int f d x=\int_{\mathbb{R}^{3}} f d x
$$

For $1 \leq r \leq \infty$ and $\beta>0$, we denote the standard homogeneous and inhomogeneous Sobolev spaces as follows:

$$
\left\{\begin{array}{l}
L^{r}=L^{r}\left(\mathbb{R}^{3}\right), \quad D^{k, r}=\left\{u \in L_{l o c}^{1}\left(\mathbb{R}^{3}\right) \mid\left\|\nabla^{k} u\right\|_{L^{r}}<\infty\right\}, \quad\|u\|_{D^{k, r}} \triangleq\left\|\nabla^{k} u\right\|_{L^{r}}, \\
W^{k, r}=L^{r} \cap D^{k, r}, \quad H^{k}=W^{k, 2}, \quad D^{k}=D^{k, 2}, \quad D^{1}=\left\{u \in L^{6} \mid\|\nabla u\|_{L^{2}}<\infty\right\} \\
\dot{H}^{\beta}=\left\{f:\left.\mathbb{R}^{3} \rightarrow \mathbb{R}\left|\|f\|_{\dot{H}^{\beta}}^{2}=\int\right| \xi\right|^{2 \beta}|\hat{f}(\xi)|^{2} d \xi<\infty\right\},
\end{array}\right.
$$

where $\hat{f}$ is the Fourier transform of $f$.

The initial energy is defined as:

$$
C_{0}=\int\left(\frac{1}{2} \rho_{0}\left|u_{0}\right|^{2}+G\left(\rho_{0}\right)\right) d x
$$

where $G$ denotes the potential energy density given by

$$
G(\rho) \triangleq \rho \int_{\tilde{\rho}}^{\rho} \frac{P(s)-P(\tilde{\rho})}{s^{2}} d s .
$$

It is clear that

$$
\begin{cases}G(\rho)=\frac{1}{\gamma-1} P, & \text { if } \quad \tilde{\rho}=0 \\ c_{1}(\bar{\rho}, \tilde{\rho})(\rho-\tilde{\rho})^{2} \leq G(\rho) \leq c_{2}(\bar{\rho}, \tilde{\rho})(\rho-\tilde{\rho})^{2}, & \text { if } \quad \tilde{\rho}>0,0 \leq \rho \leq \bar{\rho}\end{cases}
$$

for positive constants $c_{1}(\bar{\rho}, \tilde{\rho})$ and $c_{2}(\bar{\rho}, \tilde{\rho})$.

Then the main results in this paper can be stated as follows:

Theorem 1.1 Assume that (1.2) holds. For given numbers $M>0$ (not necessarily small), $\beta \in(1 / 2,1]$, and $\bar{\rho} \geq \tilde{\rho}+1$, suppose that the initial data $\left(\rho_{0}, u_{0}\right)$ satisfy

$$
\begin{gathered}
\rho_{0}\left|u_{0}\right|^{2}+G\left(\rho_{0}\right) \in L^{1}, \quad u_{0} \in \dot{H}^{\beta} \cap D^{1} \cap D^{3}, \quad\left(\rho_{0}-\tilde{\rho}, P\left(\rho_{0}\right)-P(\tilde{\rho})\right) \in H^{3}, \\
0 \leq \inf \rho_{0} \leq \sup \rho_{0} \leq \bar{\rho}, \quad\left\|u_{0}\right\|_{\dot{H}^{\beta}} \leq M,
\end{gathered}
$$

and the compatibility condition

$$
-\mu \triangle u_{0}-(\mu+\lambda) \nabla \operatorname{divu}_{0}+\nabla P\left(\rho_{0}\right)=\rho_{0} g,
$$

for some $g \in D^{1}$ with $\rho_{0}^{1 / 2} g \in L^{2}$. Then there exists a positive constant $\varepsilon$ depending on $\mu, \lambda, \tilde{\rho}, a, \gamma, \bar{\rho}, \beta$ and $M$ such that if

$$
C_{0} \leq \varepsilon
$$


the Cauchy problem (1.1) (1.3) (1.4) has a unique global classical solution $(\rho, u)$ in $\mathbb{R}^{3} \times(0, \infty)$ satisfying for any $0<\tau<T<\infty$,

$$
\begin{gathered}
0 \leq \rho(x, t) \leq 2 \bar{\rho}, \quad x \in \mathbb{R}^{3}, t \geq 0, \\
\left\{\begin{array}{l}
(\rho-\tilde{\rho}, P-P(\tilde{\rho})) \in C\left([0, T] ; H^{3}\right), \\
u \in C\left([0, T] ; D^{1} \cap D^{3}\right) \cap L^{2}\left(0, T ; D^{4}\right) \cap L^{\infty}\left(\tau, T ; D^{4}\right), \\
u_{t} \in L^{\infty}\left(0, T ; D^{1}\right) \cap L^{2}\left(0, T ; D^{2}\right) \cap L^{\infty}\left(\tau, T ; D^{2}\right) \cap H^{1}\left(\tau, T ; D^{1}\right), \\
\sqrt{\rho} u_{t} \in L^{\infty}\left(0, T ; L^{2}\right),
\end{array}\right.
\end{gathered}
$$

and the following large-time behavior:

$$
\lim _{t \rightarrow \infty} \int\left(|\rho-\tilde{\rho}|^{q}+\rho^{1 / 2}|u|^{4}+|\nabla u|^{2}\right)(x, t) d x=0,
$$

for all

$$
q \in \begin{cases}(2, \infty), & \text { for } \tilde{\rho}>0 \\ (\gamma, \infty), & \text { for } \tilde{\rho}=0\end{cases}
$$

Similar to our previous studies on the Stokes approximation equations in [25], we can obtain from (1.13) the following large time behavior of the gradient of the density when vacuum states appear initially and the far field density is away from vacuum, which is completely in contrast to the classical theory ( [15, 27]).

Theorem 1.2 In addition to the conditions of Theorem 1.1, assume further that there exists some point $x_{0} \in \mathbb{R}^{3}$ such that $\rho_{0}\left(x_{0}\right)=0$. Then if $\tilde{\rho}>0$, the unique global classical solution $(\rho, u)$ to the Cauchy problem (1.1) (1.3) (1.4) obtained in Theorem 1.1 has to blow up as $t \rightarrow \infty$, in the sense that for any $r>3$,

$$
\lim _{t \rightarrow \infty}\|\nabla \rho(\cdot, t)\|_{L^{r}}=\infty .
$$

A few remarks are in order:

Remark 1.1 The solution obtained in Theorem 1.1 becomes a classical one for positive time. Although it has small energy, yet whose oscillations could be arbitrarily large. In particular, both interior and far field vacuum states are allowed.

Remark 1.2 In the case that the far field density is away from vacuum, i.e., $\tilde{\rho}>0$, the conclusions in Theorem 1.1 generalize the classical theory of Matsumura-Nishida ( [27]) to the case of large oscillations since in this case, the requirement of small energy, (1.10), is equivalent to smallness of the mean-square norm of $\left(\rho_{0}-\tilde{\rho}, u_{0}\right)$. However, though the large-time asymptotic behavior (1.13) is similar to that in [27], yet our solution may contain vacuum states, whose appearance leads to the large time blowup behavior stated in Theorem 1.2, this is in sharp contrast to that in [15, 27] where the gradients of the density are suitably small uniformly for all time.

Remark 1.3 When the far field density is vacuum, i.e., $\tilde{\rho}=0$, the small energy assumption, (1.10), is equivalent to that both the kinetic energy and the total pressure are suitably small. There is no requirement on the size of the set of vacuum states. In 
particular, the initial density may have compact support. Thus, Theorem 1.1 can be regarded as a uniqueness and regularity theory of Lions-Feireisl's weak solutions in [7, 26] with small initial energy. It should also be noted that the conclusions in Theorem [1.1] for the case of $\tilde{\rho}=0$ are somewhat surprising since for the isentropic compressible Navier-Stokes equations (1.1), any non-trivial one-dimensional smooth solution with initial compact supported density blows up in finite time ( 34]), and the same holds true for two-dimensional smooth spherically symmetric solutions ([18]).

Remark 1.4 It should be emphasized that in Theorem 1.1, the viscosity coefficients are only assumed to satisfy the physical conditions (1.2). While the theory on weak small energy solutions, developed in [12,15], requires the additional assumption (1.5) which is crucial in establishing the time-independent upper bound for the density in the arguments in [12,15].

Remark 1.5 For the incompressible Navier-Stokes system, a lot of results on the global wellposedness in scaling invariant spaces are available [8, 22, 23]. In particular, FujitaKato [8] and Kato [22] proved that the system is globally wellposed for small initial data in the homogeneous Sobolev spaces $\dot{H}^{1 / 2}$ or in $L^{3}$. In our case, the initial energy is small, therefore, we need the boundedness assumptions on the $\dot{H}^{\beta}$-norm of the initial velocity. It should be noted here that $\dot{H}^{\beta} \hookrightarrow L^{6 /(3-2 \beta)}$ and $6 /(3-2 \beta)>3$ for $\beta>1 / 2$, which implies that, compared with the results in [8, 22], our conditions on the initial velocity may be optimal under the smallness conditions on the initial energy.

Remark 1.6 Similar ideas can be applied to study the case on bounded domain. This will be reported in a forthcoming paper [19].

We now comment on the analysis of this paper. Note that for initial data in the class satisfying (1.7)-(1.9) except $u_{0} \in \dot{H}^{\beta}$, the local existence and uniqueness of classical solutions to the Cauchy problem, (1.1)-(1.4), have been established recently in [5]. Thus, to extend the classical solution globally in time, one needs global a priori estimates on smooth solutions to (1.1)-(1.4) in suitable higher norms. Some of the main new difficulties are due to the appearance of vacuum and that there are no other constraints on the viscosity coefficients beyond the physical conditions (1.2). It turns out that the key issue in this paper is to derive both the time-independent upper bound for the density and the time-depending higher norm estimates of the smooth solution $(\rho, u)$. We start with the basic energy estimate and the initial layer analysis, and succeed in deriving an estimate on the spatial weighted $L^{3}$-norm of the velocity, the weighted spatial mean estimates on both the gradient and the material derivatives of the velocity. This is achieved by modifying the basic elegant estimates on the material derivatives of the velocity developed by Hoff ( $[10,12,13$ ) in the theory of small energy weak solutions with non-vacuum far fields and an interpolation argument. Then we are able to obtain the desired estimates on $L^{1}\left(0, \min \{1, T\} ; L^{\infty}\left(\mathbb{R}^{3}\right)\right)$-norm and the time-independent ones on $L^{8 / 3}\left(\min \{1, T\}, T ; L^{\infty}\left(\mathbb{R}^{3}\right)\right)$-norm of the effective viscous flux (see (2.5) for the definition). It follows from these key estimates and Zlotnik's inequality (see Lemma 2.4) that the density admits a time-uniform upper bound which is the key for global estimates of classical solutions. This approach to estimate a uniform upper bound for the density is motivated by our previous analysis on the two-dimensional Stokes approximation equations in [25. The next main step is to bound the gradients of the density and the velocity. Motivated by our recent studies ( $[16,17,20]$ ) on the blow-up criteria of 
classical (or strong) solutions to (1.1), such bounds can be obtained by solving a logarithm Gronwall inequality based on a Beal-Kato-Majda type inequality (see Lemma 2.5) and the a priori estimates we have just derived, and moreover, such a derivation yields simultaneously also the bound for $L^{1}\left(0, T ; L^{\infty}\left(\mathbb{R}^{3}\right)\right)$-norm of the gradient of the velocity, see Lemma 3.6 and its proof. It should be noted here that we do not require smallness of the gradient of the initial density which prevents the appearance of vacuum $([15,27])$. Finally, with these a priori estimates on the gradients of the density and the velocity at hand, one can estimate the higher order derivatives by using the same arguments as in [20] to obtain the desired results.

The rest of the paper is organized as follows: In Section 2, we collect some elementary facts and inequalities which will be needed in later analysis. Section 3 is devoted to deriving the necessary a priori estimates on classical solutions which are needed to extend the local solution to all time. Then finally, the main results, Theorem 1.1 and Theorem 1.2, are proved in Section 4.

\section{Preliminaries}

In this section, we will recall some known facts and elementary inequalities which will be used frequently later.

We start with the local existence and uniqueness of classical solutions when the initial density may not be positive and may vanish in an open set.

Lemma 2.1 ( [5]) For $\tilde{\rho} \geq 0$, assume that the initial data $\left(\rho_{0} \geq 0, u_{0}\right)$ satisfy (1.7)(1.9) except $u_{0} \in \dot{H}^{\beta}$. Then there exist a small time $T_{*}>0$ and a unique classical solution $(\rho, u)$ to the Cauchy problem (1.1) (1.3) 1.4) on $\mathbb{R}^{3} \times\left(0, T_{*}\right]$ such that

$$
\left\{\begin{array}{l}
(\rho-\tilde{\rho}, P-P(\tilde{\rho})) \in C\left(\left[0, T_{*}\right] ; H^{3}\right), \\
u \in C\left(\left[0, T_{*}\right] ; D^{1} \cap D^{3}\right) \cap L^{2}\left(0, T_{*} ; D^{4}\right), \\
u_{t} \in L^{\infty}\left(0, T_{*} ; D^{1}\right) \cap L^{2}\left(0, T_{*} ; D^{2}\right), \quad \sqrt{\rho} u_{t} \in L^{\infty}\left(0, T_{*} ; L^{2}\right), \\
\sqrt{\rho} u_{t t} \in L^{2}\left(0, T_{*} ; L^{2}\right), \quad t^{1 / 2} u \in L^{\infty}\left(0, T_{*} ; D^{4}\right), \\
t^{1 / 2} \sqrt{\rho} u_{t t} \in L^{\infty}\left(0, T_{*} ; L^{2}\right), \quad t u_{t} \in L^{\infty}\left(0, T_{*} ; D^{3}\right), \\
t u_{t t} \in L^{\infty}\left(0, T_{*} ; D^{1}\right) \cap L^{2}\left(0, T_{*} ; D^{2}\right) .
\end{array}\right.
$$

Next, the following well-known Gagliardo-Nirenberg inequality will be used later frequently (see [24]).

Lemma 2.2 (Gagliardo-Nirenberg) For $p \in[2,6], q \in(1, \infty)$, and $r \in(3, \infty)$, there exists some generic constant $C>0$ which may depend on $q, r$ such that for $f \in H^{1}\left(\mathbb{R}^{3}\right)$ and $g \in L^{q}\left(\mathbb{R}^{3}\right) \cap D^{1, r}\left(\mathbb{R}^{3}\right)$, we have

$$
\begin{gathered}
\|f\|_{L^{p}}^{p} \leq C\|f\|_{L^{2}}^{(6-p) / 2}\|\nabla f\|_{L^{2}}^{(3 p-6) / 2}, \\
\|g\|_{C\left(\overline{\mathbb{R}^{3}}\right)} \leq C\|g\|_{L^{q}}^{q(r-3) /(3 r+q(r-3))}\|\nabla g\|_{L^{r}}^{3 r /(3 r+q(r-3))} .
\end{gathered}
$$

We now state some elementary estimates which follow from (2.2) and the standard $L^{p}$-estimate for the following elliptic system derived from the momentum equations in (1.1):

$$
\triangle F=\operatorname{div}(\rho \dot{u}), \quad \mu \triangle \omega=\nabla \times(\rho \dot{u}),
$$


where

$$
\dot{f} \triangleq f_{t}+u \cdot \nabla f, \quad F \triangleq(2 \mu+\lambda) \operatorname{div} u-P(\rho)+P(\tilde{\rho}), \quad \omega \triangleq \nabla \times u,
$$

are the material derivative of $f$, the effective viscous flux and the vorticity respectively.

Lemma 2.3 Let $(\rho, u)$ be a smooth solution of (1.1) (1.3). Then there exists a generic positive constant $C$ depending only on $\mu$ and $\lambda$ such that for any $p \in[2,6]$

$$
\begin{aligned}
& \|\nabla F\|_{L^{p}}+\|\nabla \omega\|_{L^{p}} \leq C\|\rho \dot{u}\|_{L^{p}} \\
& \|F\|_{L^{p}}+\|\omega\|_{L^{p}} \leq C\|\rho \dot{u}\|_{L^{2}}^{(3 p-6) /(2 p)}\left(\|\nabla u\|_{L^{2}}+\|P-P(\tilde{\rho})\|_{L^{2}}\right)^{(6-p) /(2 p)} \\
& \|\nabla u\|_{L^{p}} \leq C\left(\|F\|_{L^{p}}+\|\omega\|_{L^{p}}\right)+C\|P-P(\tilde{\rho})\|_{L^{p}} \\
& \|\nabla u\|_{L^{p}} \leq C\|\nabla u\|_{L^{2}}^{(6-p) /(2 p)}\left(\|\rho \dot{u}\|_{L^{2}}+\|P-P(\tilde{\rho})\|_{L^{6}}\right)^{(3 p-6) /(2 p)}
\end{aligned}
$$

Proof. The standard $L^{p}$-estimate for the elliptic system (2.4) yields directly (2.6), which, together with (2.2) and (2.5), gives (2.7).

Note that $-\Delta u=-\nabla \operatorname{div} u+\nabla \times \omega$, which implies that

$$
\nabla u=-\nabla(-\Delta)^{-1} \nabla \operatorname{div} u+\nabla(-\Delta)^{-1} \nabla \times \omega .
$$

Thus the standard $L^{p}$ estimate shows that

$$
\|\nabla u\|_{L^{p}} \leq C\left(\|\operatorname{div} u\|_{L^{p}}+\|\omega\|_{L^{p}}\right), \text { for } p \in[2,6],
$$

which, together with (2.5), gives (2.8). Now (2.9) follows from (2.2), (2.8) and (2.6).

Next, the following Zlotnik inequality will be used to get the uniform (in time) upper bound of the density $\rho$.

Lemma 2.4 ( [35]) Let the function y satisfy

$$
y^{\prime}(t)=g(y)+b^{\prime}(t) \text { on }[0, T], \quad y(0)=y^{0},
$$

with $g \in C(R)$ and $y, b \in W^{1,1}(0, T)$. If $g(\infty)=-\infty$ and

$$
b\left(t_{2}\right)-b\left(t_{1}\right) \leq N_{0}+N_{1}\left(t_{2}-t_{1}\right)
$$

for all $0 \leq t_{1}<t_{2} \leq T$ with some $N_{0} \geq 0$ and $N_{1} \geq 0$, then

$$
y(t) \leq \max \left\{y^{0}, \bar{\zeta}\right\}+N_{0}<\infty \text { on }[0, T],
$$

where $\bar{\zeta}$ is a constant such that

$$
g(\zeta) \leq-N_{1} \quad \text { for } \quad \zeta \geq \bar{\zeta}
$$

Finally, we state the following Beal-Kato-Majda type inequality which was proved in [1] when $\operatorname{div} u \equiv 0$ and will be used later to estimate $\|\nabla u\|_{L^{\infty}}$ and $\|\nabla \rho\|_{L^{2} \cap L^{6}}$.

Lemma 2.5 For $3<q<\infty$, there is a constant $C(q)$ such that the following estimate holds for all $\nabla u \in L^{2}\left(\mathbb{R}^{3}\right) \cap D^{1, q}\left(\mathbb{R}^{3}\right)$,

$$
\begin{aligned}
\|\nabla u\|_{L^{\infty}\left(\mathbb{R}^{3}\right)} \leq & C\left(\|\operatorname{div} u\|_{L^{\infty}\left(\mathbb{R}^{3}\right)}+\|\omega\|_{L^{\infty}\left(\mathbb{R}^{3}\right)}\right) \log \left(e+\left\|\nabla^{2} u\right\|_{L^{q}\left(\mathbb{R}^{3}\right)}\right) \\
& +C\|\nabla u\|_{L^{2}\left(\mathbb{R}^{3}\right)}+C .
\end{aligned}
$$


Proof. The proof is similar to that of (15) in [1] and is sketched here for completeness. It follows from the Poisson's formula that

$$
\begin{aligned}
u(x) & =-\frac{1}{4 \pi} \int \frac{\Delta u(y)}{|x-y|} d y \\
& \equiv \int \operatorname{div} u(y) K(x-y) d y-\int K(x-y) \times \omega(y) d y \\
& \triangleq v+w,
\end{aligned}
$$

where

$$
K(x-y) \triangleq \frac{x-y}{4 \pi|x-y|^{3}}
$$

satisfies

$$
|K(x-y) \leq C| x-\left.y\right|^{-2}, \quad|\nabla K(x-y)| \leq C|x-y|^{-3} .
$$

It suffices to estimate the term $\nabla v$ since $\nabla w$ can be handled similarly (see [1]). Let $\delta \in(0,1]$ be a constant to be chosen and introduce a cut-off function $\eta_{\delta}(x)$ satisfying $\eta_{\delta}(x)=1$ for $|x|<\delta, \eta_{\delta}(x)=0$ for $|x|>2 \delta$, and $\left|\nabla \eta_{\delta}(x)\right| \leq C \delta^{-1}$. Then $\nabla v$ can be rewritten as

$$
\begin{aligned}
\nabla v= & \int \eta_{\delta}(y) K(y) \nabla \operatorname{div} u(x-y) d y-\int \nabla \eta_{\delta}(x-y) K(x-y) \operatorname{div} u(y) d y \\
& +\int\left(1-\eta_{\delta}(x-y)\right) \nabla K(x-y) \operatorname{div} u(y) d y
\end{aligned}
$$

Each term on the righthand side of (2.14) can be estimated by (2.13) as follows:

$$
\begin{gathered}
\left|\int \eta_{\delta}(y) K(y) \nabla \operatorname{div} u(x-y) d y\right| \\
\leq C\left\|\eta_{\delta}(y) K(y)\right\|_{L^{q /(q-1)}}\left\|\nabla^{2} u\right\|_{L^{q}} \\
\leq C\left(\int_{0}^{2 \delta} r^{-2 q /(q-1)} r^{2} d r\right)^{(q-1) / q}\left\|\nabla^{2} u\right\|_{L^{q}} \\
\leq C \delta^{(q-3) / q}\left\|\nabla^{2} u\right\|_{L^{q}}, \\
\quad\left|\int \nabla \eta_{\delta}(x-y) K(x-y) \operatorname{div} u(y) d y\right| \\
\leq \int\left|\nabla \eta_{\delta}(z)\|K(z) \mid d z\| \operatorname{div} u \|_{L^{\infty}}\right. \\
\leq C \int_{\delta}^{2 \delta} \delta^{-1} r^{-2} r^{2} d r\|\operatorname{div} u\|_{L^{\infty}} \\
\leq C\|\operatorname{div} u\|_{L^{\infty}}, \\
\left|\int\left(1-\eta_{\delta}(x-y)\right) \nabla K(x-y) \operatorname{div} u(y) d y\right| \\
\leq C\left(\int_{\delta \leq|x-y| \leq 1}+\int_{|x-y|>1}|\nabla K(x-y) \| \operatorname{div} u(y)| d y\right. \\
\leq C \int_{\delta}^{1} r^{-3} r^{2} d r\|\operatorname{div} u\|_{L^{\infty}}+C\left(\int_{1}^{\infty} r^{-6} r^{2} d r\right)^{1 / 2}\|\operatorname{div} u\|_{L^{2}} \\
\leq-C \ln \delta\|\operatorname{div} u\|_{L^{\infty}}+C\|\nabla u\|_{L^{2}}
\end{gathered}
$$


It follows from (2.14)-(2.17) that

$$
\|\nabla v\|_{L^{\infty}} \leq C\left(\delta^{(q-3) / q}\left\|\nabla^{2} u\right\|_{L^{q}}+(1-\ln \delta)\|\operatorname{div} u\|_{L^{\infty}}+\|\nabla u\|_{L^{2}}\right) .
$$

Set $\delta=\min \left\{1,\left\|\nabla^{2} u\right\|_{L^{q}}^{-q /(q-3)}\right\}$. Then (2.18) becomes

$$
\|\nabla v\|_{L^{\infty}} \leq C(q)\left(1+\ln \left(e+\left\|\nabla^{2} u\right\|_{L^{q}}\right)\|\operatorname{div} u\|_{L^{\infty}}+\|\nabla u\|_{L^{2}}\right) .
$$

Therefore (2.12) holds.

\section{A priori estimates}

In this section, we will establish some necessary a priori bounds for smooth solutions to the Cauchy problem (1.1) (1.3) (1.4) to extend the local classical solution guaranteed by Lemma 2.1. Thus, let $T>0$ be a fixed time and $(\rho, u)$ be the smooth solution to (1.1) (1.3) (1.4) on $\mathbb{R}^{3} \times(0, T]$ in the class (2.1) with smooth initial data $\left(\rho_{0}, u_{0}\right)$ satisfying (1.7)-(1.9). To estimate this solution, we set $\sigma(t) \triangleq \min \{1, t\}$ and define

$$
\begin{gathered}
A_{1}(T) \triangleq \sup _{t \in[0, T]}\left(\sigma\|\nabla u\|_{L^{2}}^{2}\right)+\int_{0}^{T} \int \sigma \rho|\dot{u}|^{2} d x d t, \\
A_{2}(T) \triangleq \sup _{t \in[0, T]} \sigma^{3} \int \rho|\dot{u}|^{2} d x+\int_{0}^{T} \int \sigma^{3}|\nabla \dot{u}|^{2} d x d t,
\end{gathered}
$$

and

$$
A_{3}(T) \triangleq \sup _{0 \leq t \leq T} \int \rho|u|^{3}(x, t) d x .
$$

We have the following key a priori estimates on $(\rho, u)$.

Proposition 3.1 Under the conditions of Theorem 1.1, for

$$
\delta_{0} \triangleq(2 \beta-1) /(4 \beta) \in(0,1 / 4],
$$

there exists some positive constant $\varepsilon$ depending on $\mu, \lambda, \tilde{\rho}, a, \gamma, \bar{\rho}, \beta$ and $M$ such that if $(\rho, u)$ is a smooth solution of (1.1) (1.3) 1.4) on $\mathbb{R}^{3} \times(0, T]$ satisfying

$$
\sup _{\mathbb{R}^{3} \times[0, T]} \rho \leq 2 \bar{\rho}, \quad A_{1}(T)+A_{2}(T) \leq 2 C_{0}^{1 / 2}, \quad A_{3}(\sigma(T)) \leq 2 C_{0}^{\delta_{0}},
$$

the following estimates hold

$$
\sup _{\mathbb{R}^{3} \times[0, T]} \rho \leq 7 \bar{\rho} / 4, \quad A_{1}(T)+A_{2}(T) \leq C_{0}^{1 / 2}, \quad A_{3}(\sigma(T)) \leq C_{0}^{\delta_{0}},
$$

provided $C_{0} \leq \varepsilon$.

Proof. Proposition 3.1 is an easy consequence of the following Lemmas 3.3, 3.4 and 3.5 .

In the following, we will use the convention that $C$ denotes a generic positive constant depending on $\mu, \lambda, \tilde{\rho}, a, \gamma, \bar{\rho}, \beta$ and $M$, and we write $C(\alpha)$ to emphasize that $C$ depends on $\alpha$.

We start with the following standard energy estimate for $(\rho, u)$ and preliminary $L^{2}$ bounds for $\nabla u$ and $\rho \dot{u}$. 
Lemma 3.1 Let $(\rho, u)$ be a smooth solution of (1.1) (1.3) (1.4) on $\mathbb{R}^{3} \times(0, T]$ with $0 \leq \rho(x, t) \leq 2 \bar{\rho}$. Then there is a positive constant $C=C(\bar{\rho})$ such that

$$
\begin{gathered}
\sup _{0 \leq t \leq T} \int\left(\frac{1}{2} \rho|u|^{2}+G(\rho)\right) d x+\int_{0}^{T} \int\left(\mu|\nabla u|^{2}+(\lambda+\mu)(\operatorname{div} u)^{2}\right) d x d t \leq C_{0}, \\
A_{1}(T) \leq C C_{0}+C \int_{0}^{T} \int \sigma|\nabla u|^{3} d x d t
\end{gathered}
$$

and

$$
A_{2}(T) \leq C C_{0}+C A_{1}(T)+C \int_{0}^{T} \int \sigma^{3}|\nabla u|^{4} d x d t .
$$

Proof. Multiplying the first equation in (1.1) by $G^{\prime}(\rho)$ and the second by $u^{j}$ and integrating, applying the far field condition (1.3), one shows easily the energy inequality (3.6) .

The proof of (3.7) and (3.8) is due to Hoff [10]. For $m \geq 0$, multiplying (1.1) 2 by $\sigma^{m} \dot{u}$ and then integrating the resulting equality over $\mathbb{R}^{3}$ lead to

$$
\begin{aligned}
\int \sigma^{m} \rho|\dot{u}|^{2} d x & =\int\left(-\sigma^{m} \dot{u} \cdot \nabla P+\mu \sigma^{m} \triangle u \cdot \dot{u}+(\lambda+\mu) \sigma^{m} \nabla \operatorname{div} u \cdot \dot{u}\right) d x \\
& \triangleq \sum_{i=1}^{3} M_{i} .
\end{aligned}
$$

Using (1.1) 1 and integrating by parts give

$$
\begin{aligned}
M_{1}= & -\int \sigma^{m} \dot{u} \cdot \nabla P d x \\
= & \int\left(\sigma^{m}(\operatorname{div} u)_{t}(P-P(\tilde{\rho}))-\sigma^{m}(u \cdot \nabla u) \cdot \nabla P\right) d x \\
= & \left(\int \sigma^{m} \operatorname{div} u(P-P(\tilde{\rho})) d x\right)_{t}-m \sigma^{m-1} \sigma^{\prime} \int \operatorname{div} u(P-P(\tilde{\rho})) d x \\
& +\int \sigma^{m}\left(P^{\prime} \rho(\operatorname{div} u)^{2}-P(\operatorname{div} u)^{2}+P \partial_{i} u^{j} \partial_{j} u^{i}\right) d x \\
\leq & \left(\int \sigma^{m} \operatorname{div} u(P-P(\tilde{\rho})) d x\right)_{t}+m \sigma^{m-1} \sigma^{\prime}\|P-P(\tilde{\rho})\|_{L^{2}}\|\nabla u\|_{L^{2}} \\
& +C(\bar{\rho})\|\nabla u\|_{L^{2}}^{2} \\
\leq & \left(\int \sigma^{m} \operatorname{div} u(P-P(\tilde{\rho})) d x\right)_{t}+C(\bar{\rho})\|\nabla u\|_{L^{2}}^{2}+C(\bar{\rho}) m^{2} \sigma^{2(m-1)} \sigma^{\prime} C_{0} .
\end{aligned}
$$

Integration by parts implies

$$
\begin{aligned}
M_{2} & =\int \mu \sigma^{m} \triangle u \cdot \dot{u} d x \\
& =-\frac{\mu}{2}\left(\sigma^{m}\|\nabla u\|_{L^{2}}^{2}\right)_{t}+\frac{\mu m}{2} \sigma^{m-1} \sigma^{\prime}\|\nabla u\|_{L^{2}}^{2}-\mu \sigma^{m} \int \partial_{i} u^{j} \partial_{i}\left(u^{k} \partial_{k} u^{j}\right) d x \\
& \leq-\frac{\mu}{2}\left(\sigma^{m}\|\nabla u\|_{L^{2}}^{2}\right)_{t}+C m \sigma^{m-1}\|\nabla u\|_{L^{2}}^{2}+C \int \sigma^{m}|\nabla u|^{3} d x
\end{aligned}
$$


and similarly,

$$
\begin{aligned}
M_{3} & =-\frac{\lambda+\mu}{2}\left(\sigma^{m}\|\operatorname{div} u\|_{L^{2}}^{2}\right)_{t}+\frac{m(\lambda+\mu)}{2} \sigma^{m-1}\|\operatorname{div} u\|_{L^{2}}^{2} \\
& -(\lambda+\mu) \sigma^{m} \int \operatorname{div} u \operatorname{div}(u \cdot \nabla u) d x \\
& \leq-\frac{\lambda+\mu}{2}\left(\sigma^{m}\|\operatorname{div} u\|_{L^{2}}^{2}\right)_{t}+C m \sigma^{m-1}\|\nabla u\|_{L^{2}}^{2}+C \int \sigma^{m}|\nabla u|^{3} d x .
\end{aligned}
$$

Combining (3.9)-(3.12) leads to

$$
\begin{aligned}
& \left(\sigma^{m} B(t)\right)^{\prime}+\int \sigma^{m} \rho|\dot{u}|^{2} d x \\
& \leq\left(C m \sigma^{m-1}+C(\bar{\rho})\right)\|\nabla u\|_{L^{2}}^{2}+C(\bar{\rho}) m^{2} \sigma^{2(m-1)} \sigma^{\prime} C_{0}+C \int \sigma^{m}|\nabla u|^{3} d x,
\end{aligned}
$$

where

$$
\begin{aligned}
B(t) & \triangleq \frac{\mu}{2}\|\nabla u\|_{L^{2}}^{2}+\frac{(\lambda+\mu)}{2}\|\operatorname{div} u\|_{L^{2}}^{2}-\int \operatorname{div} u(P-P(\tilde{\rho})) d x \\
& \geq \frac{\mu}{2}\|\nabla u\|_{L^{2}}^{2}+\frac{(\lambda+\mu)}{2}\|\operatorname{div} u\|_{L^{2}}^{2}-C C_{0}^{1 / 2}\|\operatorname{div} u\|_{L^{2}} \\
& \geq \frac{\mu}{4}\|\nabla u\|_{L^{2}}^{2}+\frac{(\lambda+\mu)}{2}\|\operatorname{div} u\|_{L^{2}}^{2}-C C_{0} .
\end{aligned}
$$

Integrating (3.13) over $(0, T)$, choosing $m=1$, and using (3.14), one gets (3.7).

Next, for $m \geq 0$, operating $\sigma^{m} \dot{u}^{j}[\partial / \partial t+\operatorname{div}(u \cdot)]$ to $(1.1)_{2}^{j}$, summing with respect to $j$, and integrating the resulting equation over $\mathbb{R}^{3}$, one obtains after integration by parts

$$
\begin{aligned}
& \left(\frac{\sigma^{m}}{2} \int \rho|\dot{u}|^{2} d x\right)_{t}-\frac{m}{2} \sigma^{m-1} \sigma^{\prime} \int \rho|\dot{u}|^{2} d x \\
& =-\int \sigma^{m} \dot{u}^{j}\left[\partial_{j} P_{t}+\operatorname{div}\left(\partial_{j} P u\right)\right] d x+\mu \int \sigma^{m} \dot{u}^{j}\left[\triangle u_{t}^{j}+\operatorname{div}\left(u \triangle u^{j}\right)\right] d x \\
& \quad+(\lambda+\mu) \int \sigma^{m} \dot{u}^{j}\left[\partial_{t} \partial_{j} \operatorname{div} u+\operatorname{div}\left(u \partial_{j} \operatorname{div} u\right)\right] d x \\
& \triangleq \sum_{i=1}^{3} N_{i} .
\end{aligned}
$$

It follows from integration by parts and using the equation (1.1) 1 that

$$
\begin{aligned}
N_{1} & =-\int \sigma^{m} \dot{u}^{j}\left[\partial_{j} P_{t}+\operatorname{div}\left(\partial_{j} P u\right)\right] d x \\
& =\int \sigma^{m}\left[-P^{\prime} \rho \operatorname{div} u \partial_{j} \dot{u}^{j}+\partial_{k}\left(\partial_{j} \dot{u}^{j} u^{k}\right) P-P \partial_{j}\left(\partial_{k} \dot{u}^{j} u^{k}\right)\right] d x \\
& \leq C(\bar{\rho}) \sigma^{m}\|\nabla u\|_{L^{2}}\|\nabla \dot{u}\|_{L^{2}} \\
& \leq \delta \sigma^{m}\|\nabla \dot{u}\|_{L^{2}}^{2}+C(\bar{\rho}, \delta) \sigma^{m}\|\nabla u\|_{L^{2}}^{2} .
\end{aligned}
$$

Integration by parts leads to

$$
\begin{aligned}
N_{2} & =\mu \int \sigma^{m} \dot{u}^{j}\left[\triangle u_{t}^{j}+\operatorname{div}\left(u \triangle u^{j}\right)\right] d x \\
& =-\mu \int \sigma^{m}\left[|\nabla \dot{u}|^{2}+\partial_{i} \dot{u}^{j} \partial_{k} u^{k} \partial_{i} u^{j}-\partial_{i} \dot{u}^{j} \partial_{i} u^{k} \partial_{k} u^{j}-\partial_{i} u^{j} \partial_{i} u^{k} \partial_{k} \dot{u}^{j}\right] d x \\
& \leq-\frac{3 \mu}{4} \int \sigma^{m}|\nabla \dot{u}|^{2} d x+C \int \sigma^{m}|\nabla u|^{4} d x .
\end{aligned}
$$


Similarly,

$$
N_{3} \leq-\frac{\mu+\lambda}{2} \int \sigma^{m}(\operatorname{div} \dot{u})^{2} d x+C \int \sigma^{m}|\nabla u|^{4} d x .
$$

Substituting (3.16)-(3.18) into (3.15) shows that for $\delta$ suitably small, it holds that

$$
\begin{aligned}
& \left(\sigma^{m} \int \rho|\dot{u}|^{2} d x\right)_{t}+\mu \int \sigma^{m}|\nabla \dot{u}|^{2} d x+(\mu+\lambda) \int \sigma^{m}(\operatorname{div} \dot{u})^{2} d x \\
& \leq m \sigma^{m-1} \sigma^{\prime} \int \rho|\dot{u}|^{2} d x+C \sigma^{m}\|\nabla u\|_{L^{4}}^{4}+C(\bar{\rho}) \sigma^{m}\|\nabla u\|_{L^{2}}^{2} .
\end{aligned}
$$

Taking $m=3$ in (3.19) and noticing that

$$
3 \int_{0}^{T} \sigma^{2} \sigma^{\prime} \int \rho|\dot{u}|^{2} d x d t \leq C A_{1}(T)
$$

we immediately obtain (3.8) after integrating (3.19) over $(0, T)$. The proof of Lemma 3.1 is completed.

Next, the following lemma will play important roles in the estimates on both $A_{i}(\sigma(T))$ $(i=1,3)$ and the uniform upper bound of the density for small time.

Lemma 3.2 Let $(\rho, u)$ be a smooth solution of (1.1) (1.3) (1.4) on $\mathbb{R}^{3} \times(0, T]$ satisfying (3.4). Then there exist positive constants $K$ and $\varepsilon_{0}$ both depending only on $\mu, \lambda, \tilde{\rho}, a$, $\gamma, \bar{\rho}, \beta$ and $M$ such that

$$
\begin{gathered}
\sup _{0 \leq t \leq \sigma(T)} t^{1-\beta}\|\nabla u\|_{L^{2}}^{2}+\int_{0}^{\sigma(T)} t^{1-\beta} \int \rho|\dot{u}|^{2} d x d t \leq K(\bar{\rho}, M), \\
\sup _{0 \leq t \leq \sigma(T)} t^{2-\beta} \int \rho|\dot{u}|^{2} d x+\int_{0}^{\sigma(T)} t^{2-\beta} \int|\nabla \dot{u}|^{2} d x d t \leq K(\bar{\rho}, M),
\end{gathered}
$$

provided $C_{0} \leq \varepsilon_{0}$.

Proof. As in [13, we define $w_{1}$ and $w_{2}$ to be the solution to:

$$
L w_{1}=0, \quad w_{1}(x, 0)=w_{10}(x)
$$

and

$$
L w_{2}=-\nabla P(\rho), \quad w_{2}(x, 0)=0,
$$

respectively, with $L$ being the linear differential operator defined by

$$
\begin{aligned}
(L w)^{j} & \triangleq \rho w_{t}^{j}+\rho u \cdot \nabla w^{j}-\left(\mu \Delta w^{j}+(\mu+\lambda) \operatorname{div} w_{x_{j}}\right) \\
& =\rho \dot{w}^{j}-\left(\mu \Delta w^{j}+(\mu+\lambda) \operatorname{div} w_{x_{j}}\right), \quad j=1,2,3 .
\end{aligned}
$$

Straightforward energy estimates show that:

$$
\sup _{0 \leq t \leq \sigma(T)} \int \rho\left|w_{1}\right|^{2} d x+\int_{0}^{\sigma(T)} \int\left|\nabla w_{1}\right|^{2} d x d t \leq C(\bar{\rho}) \int\left|w_{10}\right|^{2} d x
$$

and

$$
\sup _{0 \leq t \leq \sigma(T)} \int \rho\left|w_{2}\right|^{2} d x+\int_{0}^{\sigma(T)} \int\left|\nabla w_{2}\right|^{2} d x d t \leq C(\bar{\rho}) C_{0}
$$


It follows from (3.22) and standard $L^{2}$-estimate for elliptic system that

$$
\left\|\nabla w_{1}\right\|_{L^{6}} \leq C\left\|\nabla^{2} w_{1}\right\|_{L^{2}} \leq C\left\|\rho \dot{w}_{1}\right\|_{L^{2}} .
$$

Multiplying (3.22) by $w_{1 t}$ and integrating the resulting equality over $\mathbb{R}^{3}$, we get by (3.26) and (3.4) 3 that

$$
\begin{aligned}
& \frac{1}{2}\left(\mu\left\|\nabla w_{1}\right\|_{L^{2}}^{2}+(\mu+\lambda)\left\|\operatorname{div} w_{1}\right\|_{L^{2}}^{2}\right)_{t}+\int \rho\left|\dot{w}_{1}\right|^{2} d x \\
& =\int \rho \dot{w}_{1}\left(u \cdot \nabla w_{1}\right) d x \\
& \leq C(\bar{\rho})\left(\int \rho\left|\dot{w}_{1}\right|^{2} d x\right)^{1 / 2}\left(\int \rho|u|^{3} d x\right)^{1 / 3}\left\|\nabla w_{1}\right\|_{L^{6}} \\
& \leq C(\bar{\rho}) C_{0}^{\delta_{0} / 3} \int \rho\left|\dot{w}_{1}\right|^{2} d x
\end{aligned}
$$

which, together with Gronwall's inequality and (3.24), gives

$$
\sup _{0 \leq t \leq \sigma(T)}\left\|\nabla w_{1}\right\|_{L^{2}}^{2}+\int_{0}^{\sigma(T)} \int \rho\left|\dot{w}_{1}\right|^{2} d x d t \leq C\left\|\nabla w_{10}\right\|_{L^{2}}^{2},
$$

and

$$
\sup _{0 \leq t \leq \sigma(T)} t\left\|\nabla w_{1}\right\|_{L^{2}}^{2}+\int_{0}^{\sigma(T)} t \int \rho\left|\dot{w}_{1}\right|^{2} d x d t \leq C\left\|w_{10}\right\|_{L^{2}}^{2}
$$

provided $C_{0} \leq \varepsilon_{01} \triangleq(2 C(\bar{\rho}))^{-3 / \delta_{0}}$.

Since the solution operator $w_{10} \mapsto w_{1}(\cdot, t)$ is linear, by the standard Stein-Weiss interpolation argument ( [2]), one can deduce from (3.27) and (3.28) that for any $\theta \in[\beta, 1]$,

$$
\sup _{0 \leq t \leq \sigma(T)} t^{1-\theta}\left\|\nabla w_{1}\right\|_{L^{2}}^{2}+\int_{0}^{\sigma(T)} t^{1-\theta} \int \rho\left|\dot{w}_{1}\right|^{2} d x d t \leq C\left\|w_{10}\right\|_{\dot{H}^{\theta}}^{2},
$$

with a uniform constant $C$ independent of $\theta$.

Next, we estimate $w_{2}$. It follows from a similar way to (2.6) and (2.8) that

$$
\left\{\begin{array}{l}
\left\|\nabla\left((2 \mu+\lambda) \operatorname{div} w_{2}-(P-P(\tilde{\rho}))\right)\right\|_{L^{2}} \leq C\left\|\rho \dot{w}_{2}\right\|_{L^{2}}, \\
\left\|\nabla w_{2}\right\|_{L^{6}} \leq C\left(\left\|\rho \dot{w}_{2}\right\|_{L^{2}}+\|P-P(\tilde{\rho})\|_{L^{6}}\right) .
\end{array}\right.
$$

Multiplying (‥23) by $w_{2 t}$, integrating the resultant equation over $\mathbb{R}^{3}$ and using (3.30), 
one has

$$
\begin{aligned}
\frac{1}{2} & \left(\mu\left\|\nabla w_{2}\right\|_{L^{2}}^{2}+(\mu+\lambda)\left\|\operatorname{div} w_{2}\right\|_{L^{2}}^{2}-2 \int(P-P(\tilde{\rho})) \operatorname{div} w_{2} d x\right)_{t}+\int \rho\left|\dot{w}_{2}\right|^{2} d x \\
= & \int \rho \dot{w}_{2}\left(u \cdot \nabla w_{2}\right) d x-\int P_{t} \operatorname{div} w_{2} d x \\
\leq & C(\bar{\rho})\left(\int \rho\left|\dot{w}_{2}\right|^{2} d x\right)^{1 / 2}\left(\int \rho|u|^{3} d x\right)^{1 / 3}\left\|\nabla w_{2}\right\|_{L^{6}} \\
& +\int \operatorname{div} w_{2} \operatorname{div}((P-P(\tilde{\rho})) u) d x+\int(P(\tilde{\rho})+(\gamma-1) P) \operatorname{div} u \operatorname{div} w_{2} d x \\
\leq & C(\bar{\rho}) C_{0}^{\delta_{0} / 3}\left(\int \rho\left|\dot{w}_{2}\right|^{2} d x\right)^{1 / 2}\left(\left\|\rho^{1 / 2} \dot{w}_{2}\right\|_{L^{2}}+\|P-P(\tilde{\rho})\|_{L^{6}}\right) \\
& -\int(P-P(\tilde{\rho})) u \cdot \nabla\left(\operatorname{div} w_{2}-\frac{P-P(\tilde{\rho})}{2 \mu+\lambda}\right) d x \\
& +\frac{1}{2(2 \mu+\lambda)} \int(P-P(\tilde{\rho}))^{2} \operatorname{div} u d x+C\|\nabla u\|_{L^{2}}^{2}+C\left\|\nabla w_{2}\right\|_{L^{2}}^{2} \\
\leq & C(\bar{\rho}) C_{0}^{\delta_{0} / 3} \int \rho\left|\dot{w}_{2}\right|^{2} d x+C C_{0}^{1 / 3}+C\|P-P(\tilde{\rho})\|_{L^{3}}\|u\|_{L^{6}}\left\|\rho^{1 / 2} \dot{w}_{2}\right\|_{L^{2}} \\
& +C\|P-P(\tilde{\rho})\|_{L^{4}}^{4}+C\|\nabla u\|_{L^{2}}^{2}+C\left\|\nabla w_{2}\right\|_{L^{2}}^{2} \\
\leq & C(\bar{\rho}) C_{0}^{\delta_{0} / 3} \int \rho\left|\dot{w}_{2}\right|^{2} d x+C C_{0}^{1 / 3}+C\|\nabla u\|_{L^{2}}^{2}+C\left\|\nabla w_{2}\right\|_{L^{2}}^{2},
\end{aligned}
$$

which, together with (3.25) and Gronwall's inequality, gives

$$
\sup _{0 \leq t \leq \sigma(T)}\left\|\nabla w_{2}\right\|_{L^{2}}^{2}+\int_{0}^{\sigma(T)} \int \rho\left|\dot{w}_{2}\right|^{2} d x d t \leq C C_{0}^{1 / 3},
$$

provided $C_{0} \leq \varepsilon_{02} \triangleq(2 C(\bar{\rho}))^{-3 / \delta_{0}}$. Taking $w_{10}=u_{0}$ so that $w_{1}+w_{2}=u$, we then conclude from (3.29) and (3.31) that for any $\theta \in[\beta, 1]$,

$$
\sup _{0 \leq t \leq \sigma(T)} t^{1-\theta}\|\nabla u\|_{L^{2}}^{2}+\int_{0}^{\sigma(T)} t^{1-\theta} \int \rho|\dot{u}|^{2} d x d t \leq C\left\|u_{0}\right\|_{\dot{H}^{\theta}}^{2}+C C_{0}^{1 / 3},
$$

provided $C_{0} \leq \varepsilon_{0} \triangleq \min \left\{\varepsilon_{01}, \varepsilon_{02}\right\}$. Thus, (3.20) follows from (3.32) directly.

To prove (3.21), we take $m=2-\beta$ in (3.19) to obtain, after integrating (3.19) over $(0, \sigma(T))$ and using (3.32) and (2.9), that

$$
\begin{aligned}
& \sup _{0 \leq t \leq \sigma(T)} t^{2-\beta} \int \rho|\dot{u}|^{2} d x+\int_{0}^{\sigma(T)} t^{2-\beta} \int|\nabla \dot{u}|^{2} d x d t \\
\leq & C \int_{0}^{\sigma(T)} t^{2-\beta}\|\nabla u\|_{L^{4}}^{4} d t+C(\bar{\rho}, M) \\
\leq & C \int_{0}^{\sigma(T)} t^{2-\beta}\|\nabla u\|_{L^{2}}\left(\|\rho \dot{u}\|_{L^{2}}^{3}+\|P-P(\tilde{\rho})\|_{L^{6}}^{3}\right) d t+C(\bar{\rho}, M) \\
\leq & C \int_{0}^{\sigma(T)} t^{(2 \beta-1) / 2}\left(t^{1-\beta}\|\nabla u\|_{L^{2}}^{2}\right)^{1 / 2}\left(t^{2-\beta}\left\|\rho^{1 / 2} \dot{u}\right\|_{L^{2}}^{2}\right)^{1 / 2}\left(t^{1-\beta}\left\|\rho^{1 / 2} \dot{u}\right\|_{L^{2}}^{2}\right) d t \\
& +C(\bar{\rho}, M) \\
\leq & C(\bar{\rho}, M)\left(\sup _{0 \leq t \leq \sigma(T)} t^{2-\beta} \int \rho|\dot{u}|^{2} d x\right)^{1 / 2}+C(\bar{\rho}, M),
\end{aligned}
$$


which implies (3.21). Thus, we finish the proof of Lemma 3.2.

The following Lemma 3.3 will give an estimate on $A_{3}(\sigma(T))$.

Lemma 3.3 If $(\rho, u)$ is a smooth solution of (1.1) (1.3) (1.4) on $\mathbb{R}^{3} \times(0, T]$ satisfying (3.4), there exists a positive constant $\varepsilon_{1}$ depending on $\mu, \lambda, \tilde{\rho}, a, \gamma, \bar{\rho}, \beta$ and $M$ such that the following estimate holds for $\delta_{0}$ defined by (3.3):

$$
\sup _{0 \leq t \leq \sigma(T)} \int \rho|u|^{3}(x, t) d x \leq C_{0}^{\delta_{0}}
$$

provided $C_{0} \leq \varepsilon_{1}$.

Proof. Multiplying (1.1) 2 by $3|u| u$, and integrating the resulting equation over $\mathbb{R}^{3}$, we obtain by (2.9) that

$$
\begin{aligned}
& \frac{d}{d t} \int \rho|u|^{3} d x \\
& \leq C \int\left|u \left\|\left.\nabla u\right|^{2} d x+C \int|P-P(\tilde{\rho})\|u\| \nabla u| d x\right.\right. \\
& \leq C\|u\|_{L^{6}}\|\nabla u\|_{L^{2}}^{3 / 2}\|\nabla u\|_{L^{6}}^{1 / 2}+C\|P-P(\tilde{\rho})\|_{L^{3}}\|u\|_{L^{6}}\|\nabla u\|_{L^{2}} \\
& \leq C\|\nabla u\|_{L^{2}}^{5 / 2}\left(\|\rho \dot{u}\|_{L^{2}}+\|P-P(\tilde{\rho})\|_{L^{6}}\right)^{1 / 2}+C C_{0}^{1 / 6}\|\nabla u\|_{L^{2}}^{2} \\
& \leq C\|\nabla u\|_{L^{2}}^{5 / 2}\left(\|\rho \dot{u}\|_{L^{2}}+C_{0}^{1 / 6}\right)^{1 / 2}+C C_{0}^{1 / 6}\|\nabla u\|_{L^{2}}^{2} \\
& \leq C t^{\left(2 \delta_{0}-3 / 2\right)(1-\beta)}\left(t^{1-\beta}\|\nabla u\|_{L^{2}}^{2}\right)^{-2 \delta_{0}+5 / 4}\left(t^{1-\beta}\left\|\rho^{1 / 2} \dot{u}\right\|_{L^{2}}^{2}\right)^{1 / 4}\|\nabla u\|_{L^{2}}^{4 \delta_{0}} \\
& \quad+C C_{0}^{1 / 12} t^{-3(1-\beta) / 4}\left(t^{1-\beta}\|\nabla u\|_{L^{2}}^{2}\right)^{3 / 4}\|\nabla u\|_{L^{2}}+C C_{0}^{1 / 6}\|\nabla u\|_{L^{2}}^{2},
\end{aligned}
$$

which together with (3.20) and (3.6) gives

$$
\begin{aligned}
& \sup _{0 \leq t \leq \sigma(T)} \int \rho|u|^{3} d x \\
& \leq C(\bar{\rho}, M)\left(\int_{0}^{\sigma(T)} t^{-\frac{2\left(3-4 \delta_{0}\right)(1-\beta)}{3-8 \delta_{0}}} d t\right)^{\left(3-8 \delta_{0}\right) / 4}\left(\int_{0}^{\sigma(T)}\|\nabla u\|_{L^{2}}^{2} d t\right)^{2 \delta_{0}} \\
& \quad+C(\bar{\rho}, M) C_{0}^{1 / 12}\left(\int_{0}^{\sigma(T)} t^{-3(1-\beta) / 2} d t\right)^{1 / 2}\left(\int_{0}^{\sigma(T)}\|\nabla u\|_{L^{2}}^{2} d t\right)^{1 / 2} \\
& \quad+\int \rho_{0}\left|u_{0}\right|^{3} d x+C C_{0} \\
& \leq C(\bar{\rho}, M) C_{0}^{2 \delta_{0}}
\end{aligned}
$$

provided $C_{0} \leq \varepsilon_{0}$, where in the last inequality we have used the following simple facts:

$$
\begin{aligned}
\int \rho_{0}\left|u_{0}\right|^{3} d x & \leq C\left(\int \rho_{0}\left|u_{0}\right|^{2} d x\right)^{3(2 \beta-1) /(4 \beta)}\left\|u_{0}\right\|_{\dot{H}^{\beta}}^{3 /(2 \beta)} \\
& \leq C(\bar{\rho}, M) C_{0}^{2 \delta_{0}}
\end{aligned}
$$

and

$$
\frac{2\left(3-4 \delta_{0}\right)(1-\beta)}{3-8 \delta_{0}}=1-\frac{\beta(2 \beta-1)}{2-\beta}<1
$$


due to (3.3) and $\beta \in(1 / 2,1]$. Thus, it follows from (3.34) that (3.33) holds provided $C_{0} \leq \varepsilon_{1}$, where

$$
\varepsilon_{1} \triangleq \min \left\{\varepsilon_{0},(C(\bar{\rho}, M))^{-1 / \delta_{0}}\right\}=\min \left\{\varepsilon_{0},(C(\bar{\rho}, M))^{-4 \beta /(2 \beta-1)}\right\} .
$$

The proof of Lemma 3.3 is completed.

Lemma 3.4 There exists a positive constant $\varepsilon_{2}(\mu, \lambda, \tilde{\rho}, a, \gamma, \bar{\rho}, \beta, M) \leq \varepsilon_{1}$ such that, if $(\rho, u)$ is a smooth solution of (1.1) (1.3) (1.4) on $\mathbb{R}^{3} \times(0, T]$ satisfying (3.4), then

$$
A_{1}(T)+A_{2}(T) \leq C_{0}^{1 / 2}
$$

provided $C_{0} \leq \varepsilon_{2}$.

Proof. Lemma 3.1 shows that

$$
A_{1}(T)+A_{2}(T) \leq C(\bar{\rho}) C_{0}+C(\bar{\rho}) \int_{0}^{T} \sigma^{3}\|\nabla u\|_{L^{4}}^{4} d s+C(\bar{\rho}) \int_{0}^{T} \sigma\|\nabla u\|_{L^{3}}^{3} d s .
$$

Due to (2.8),

$$
\int_{0}^{T} \sigma^{3}\|\nabla u\|_{L^{4}}^{4} d s \leq C \int_{0}^{T} \sigma^{3}\left(\|F\|_{L^{4}}^{4}+\|\omega\|_{L^{4}}^{4}\right) d s+C \int_{0}^{T} \sigma^{3}\|P-P(\tilde{\rho})\|_{L^{4}}^{4} d s .
$$

It follows from (2.7) that

$$
\begin{aligned}
& \int_{0}^{T} \sigma^{3}\left(\|F\|_{L^{4}}^{4}+\|\omega\|_{L^{4}}^{4}\right) d s \\
& \leq C \int_{0}^{T} \sigma^{3}\left(\|\nabla u\|_{L^{2}}+\|P-P(\tilde{\rho})\|_{L^{2}}\right)\|\rho \dot{u}\|_{L^{2}}^{3} d s \\
& \leq C(\bar{\rho}) \sup _{t \in(0, T]}\left(\sigma^{3 / 2}\|\sqrt{\rho} \dot{u}\|_{L^{2}}\left(\sigma^{1 / 2}\|\nabla u\|_{L^{2}}+C_{0}^{1 / 2}\right)\right) \int_{0}^{T} \int \sigma \rho|\dot{u}|^{2} d x d s \\
& \leq C(\bar{\rho})\left(A_{1}^{1 / 2}(T)+C_{0}^{1 / 2}\right) A_{2}^{1 / 2}(T) A_{1}(T) \\
& \leq C(\bar{\rho}) C_{0} .
\end{aligned}
$$

To estimate the second term on the right hand side of (3.38), one deduces from (1.1) 1 that $P-P(\tilde{\rho})$ satisfies

$$
(P-P(\tilde{\rho}))_{t}+u \cdot \nabla(P-P(\tilde{\rho}))+\gamma(P-P(\tilde{\rho})) \operatorname{div} u+\gamma P(\tilde{\rho}) \operatorname{div} u=0 .
$$

Multiplying (3.40) by $3(P-P(\tilde{\rho}))^{2}$ and integrating the resulting equality over $\mathbb{R}^{3}$, one gets after using $\operatorname{div} u=\frac{1}{2 \mu+\lambda}(F+P-P(\tilde{\rho}))$ that

$$
\begin{aligned}
\frac{3 \gamma-1}{2 \mu+\lambda} & \|P-P(\tilde{\rho})\|_{L^{4}}^{4} \\
= & -\left(\int(P-P(\tilde{\rho}))^{3} d x\right)_{t}-\frac{3 \gamma-1}{2 \mu+\lambda} \int(P-P(\tilde{\rho}))^{3} F d x \\
& -3 \gamma P(\tilde{\rho}) \int(P-P(\tilde{\rho}))^{2} \operatorname{div} u d x \\
\leq & -\left(\int(P-P(\tilde{\rho}))^{3} d x\right)_{t}+\eta\|P-P(\tilde{\rho})\|_{L^{4}}^{4}+C_{\eta}\|F\|_{L^{4}}^{4}+C_{\eta}\|\nabla u\|_{L^{2}}^{2}
\end{aligned}
$$


Multiplying (3.41) by $\sigma^{3}$, integrating the resulting inequality over $(0, T)$, and choosing $\eta$ suitably small, one may arrive at

$$
\begin{aligned}
& \int_{0}^{T} \sigma^{3}\|P-P(\tilde{\rho})\|_{L^{4}}^{4} d t \\
& \leq C \sup _{0 \leq t \leq T}\|P-P(\tilde{\rho})\|_{L^{3}}^{3}+C \int_{0}^{\sigma(T)}\|P-P(\tilde{\rho})\|_{L^{3}}^{3} d t \\
& +C(\bar{\rho}) \int_{0}^{T} \sigma^{3}\|F\|_{L^{4}}^{4} d s+C(\bar{\rho}) C_{0} \\
& \leq C(\bar{\rho}) C_{0},
\end{aligned}
$$

where (3.39) has been used. Therefore, collecting (3.38), (3.39) and (3.42) shows that

$$
\int_{0}^{T} \sigma^{3}\left(\|\nabla u\|_{L^{4}}^{4}+\|P-P(\tilde{\rho})\|_{L^{4}}^{4}\right) d s \leq C(\bar{\rho}) C_{0} .
$$

Finally, we estimate the last term on the right hand side of (3.37). First, (3.43) implies that

$$
\int_{\sigma(T)}^{T} \int \sigma|\nabla u|^{3} d x d s \leq \int_{\sigma(T)}^{T} \int\left(|\nabla u|^{4}+|\nabla u|^{2}\right) d x d s \leq C C_{0} .
$$

Next, one deduces from (2.9), (3.20) and (3.4) that

$$
\begin{aligned}
& \int_{0}^{\sigma(T)} \sigma\|\nabla u\|_{L^{3}}^{3} d t \\
& \leq C(\bar{\rho}) \int_{0}^{\sigma(T)} t\|\nabla u\|_{L^{2}}^{3 / 2}\left(\|\rho \dot{u}\|_{L^{2}}^{3 / 2}+C_{0}^{1 / 4}\right) d t \\
& \leq C(\bar{\rho}) \int_{0}^{\sigma(T)}\left(t^{(1-\beta) / 2}\|\nabla u\|_{L^{2}}\right)\|\nabla u\|_{L^{2}}^{1 / 2}\left(t \int \rho|\dot{u}|^{2} d x\right)^{3 / 4} d t+C(\bar{\rho}) C_{0} \\
& \leq C(\bar{\rho}) \sup _{t \in(0, \sigma(T)]}\left(t^{(1-\beta) / 2}\|\nabla u\|_{L^{2}}\right) \int_{0}^{\sigma(T)}\|\nabla u\|_{L^{2}}^{1 / 2}\left(t \int \rho|\dot{u}|^{2} d x\right)^{3 / 4} d t \\
& \quad+C(\bar{\rho}) C_{0} \\
& \leq C(\bar{\rho}, M) A_{1}^{3 / 4} C_{0}^{1 / 4}+C(\bar{\rho}) C_{0} \\
& \leq C(\bar{\rho}, M) C_{0}^{5 / 8}
\end{aligned}
$$

provided $C_{0} \leq \varepsilon_{1}$. It thus follows from (3.37) and (3.43) of (3.36) is bounded by

$$
C(\bar{\rho}, M) C_{0}^{5 / 8} \leq C_{0}^{1 / 2}
$$

provided

$$
C_{0} \leq \varepsilon_{2} \triangleq \min \left\{\varepsilon_{1},(C(\bar{\rho}, M))^{-8}\right\} .
$$

The proof of Lemma 3.4 is completed.

We now proceed to derive a uniform (in time) upper bound for the density, which turns out to be the key to obtain all the higher order estimates and thus to extend the classical solution globally. We will use an approach motivated by our previous study on the two-dimensional Stokes approximation equations ( [25]). 
Lemma 3.5 There exists a positive constant $\varepsilon=\varepsilon(\bar{\rho}, M)$ as described in Theorem 1.1] such that, if $(\rho, u)$ is a smooth solution of (1.1) (1.3) (1.4) on $\mathbb{R}^{3} \times(0, T]$ satisfying (3.4), then

$$
\sup _{0 \leq t \leq T}\|\rho(t)\|_{L^{\infty}} \leq \frac{7 \bar{\rho}}{4}
$$

provided $C_{0} \leq \varepsilon$.

Proof. Rewrite the equation of the mass conservation $(1.1)_{1}$ as

$$
D_{t} \rho=g(\rho)+b^{\prime}(t)
$$

where

$$
D_{t} \rho \triangleq \rho_{t}+u \cdot \nabla \rho, \quad g(\rho) \triangleq-\frac{a \rho}{2 \mu+\lambda}\left(\rho^{\gamma}-\tilde{\rho}^{\gamma}\right), \quad b(t) \triangleq-\frac{1}{2 \mu+\lambda} \int_{0}^{t} \rho F d t .
$$

For $t \in[0, \sigma(T)]$, one deduces from Lemma 2.2, (2.6), (3.36), (3.20), (3.21) and (2.3) that for $\delta_{0}$ as in (3.3) and for all $0 \leq t_{1}<t_{2} \leq \sigma(T)$,

$$
\begin{aligned}
& \left|b\left(t_{2}\right)-b\left(t_{1}\right)\right| \\
& \leq C \int_{0}^{\sigma(T)}\|(\rho F)(\cdot, t)\|_{L^{\infty}} d t \\
& \leq C(\bar{\rho}) \int_{0}^{\sigma(T)}\|F(\cdot, t)\|_{L^{6}}^{1 / 2}\|\nabla F(\cdot, t)\|_{L^{6}}^{1 / 2} d t \\
& \leq C(\bar{\rho}) \int_{0}^{\sigma(T)}\left\|\rho^{1 / 2} \dot{u}\right\|_{L^{2}}^{1 / 2}\|\nabla \dot{u}\|_{L^{2}}^{1 / 2} d t \\
& \leq C(\bar{\rho}) \int_{0}^{\sigma(T)} t^{-(2-\beta) / 4}\|\rho \dot{u}\|_{L^{2}}^{1 / 2}\left(t^{2-\beta}\|\nabla \dot{u}\|_{L^{2}}^{2}\right)^{1 / 4} d t \\
& \leq C(\bar{\rho}, M)\left(\int_{0}^{\sigma(T)} t^{-(2-\beta) / 3}\|\rho \dot{u}\|_{L^{2}}^{2 / 3} d t\right)^{3 / 4} \\
& =C(\bar{\rho}, M)\left(\int_{0}^{\sigma(T)} t^{-\left[(2-\beta)\left(-\delta_{0}+2 / 3\right)+\delta_{0}\right]}\left(t^{2-\beta}\left\|\rho^{1 / 2} \dot{u}\right\|_{L^{2}}^{2}\right)^{-\delta_{0}+1 / 3}\left(t\left\|\rho^{1 / 2} \dot{u}\right\|_{L^{2}}^{2}\right)^{\delta_{0}} d t\right)^{3 / 4} \\
& \leq C(\bar{\rho}, M)\left(A_{1}(\sigma(T))\right)^{3 \delta_{0} / 4} \\
& \leq C(\bar{\rho}, M) C_{0}^{3 \delta_{0} / 8},
\end{aligned}
$$

provided $C_{0} \leq \varepsilon_{2}$. Therefore, for $t \in[0, \sigma(T)]$, one can choose $N_{0}$ and $N_{1}$ in (2.10) as follows:

$$
N_{1}=0, \quad N_{0}=C(\bar{\rho}, M) C_{0}^{3 \delta_{0} / 8},
$$

and $\bar{\zeta}=\tilde{\rho}$ in (2.11). Then

$$
g(\zeta)=-\frac{a \zeta}{2 \mu+\lambda}\left(\zeta^{\gamma}-\tilde{\rho}^{\gamma}\right) \leq-N_{1}=0, \quad \text { for all } \quad \zeta \geq \bar{\zeta}=\tilde{\rho} .
$$

Lemma 2.4 thus yields that

$$
\sup _{t \in[0, \sigma(T)]}\|\rho\|_{L^{\infty}} \leq \max \{\bar{\rho}, \tilde{\rho}\}+N_{0} \leq \bar{\rho}+C(\bar{\rho}, M) C_{0}^{3 \delta_{0} / 8} \leq \frac{3 \bar{\rho}}{2},
$$


provided

$$
C_{0} \leq \min \left\{\varepsilon_{2}, \varepsilon_{3}\right\}, \quad \text { for } \varepsilon_{3} \triangleq\left(\frac{\bar{\rho}}{2 C(\bar{\rho}, M)}\right)^{8 /\left(3 \delta_{0}\right)}=\left(\frac{\bar{\rho}}{2 C(\bar{\rho}, M)}\right)^{32 \beta /(3(2 \beta-1))} .
$$

On the other hand, for $t \in[\sigma(T), T]$, one deduces from Lemma 2.2, (3.36), (3.6), and (2.6) that for all $\sigma(T) \leq t_{1} \leq t_{2} \leq T$,

$$
\begin{aligned}
\left|b\left(t_{2}\right)-b\left(t_{1}\right)\right| & \leq C(\bar{\rho}) \int_{t_{1}}^{t_{2}}\|F(\cdot, t)\|_{L^{\infty}} d t \\
& \leq \frac{a}{2 \mu+\lambda}\left(t_{2}-t_{1}\right)+C(\bar{\rho}) \int_{\sigma(T)}^{T}\|F(\cdot, t)\|_{L^{\infty}}^{8 / 3} d t \\
& \leq \frac{a}{2 \mu+\lambda}\left(t_{2}-t_{1}\right)+C(\bar{\rho}) \int_{\sigma(T)}^{T}\|F(\cdot, t)\|_{L^{2}}^{2 / 3}\|\nabla F(\cdot, t)\|_{L^{6}}^{2} d t \\
& \leq \frac{a}{2 \mu+\lambda}\left(t_{2}-t_{1}\right)+C(\bar{\rho}) C_{0}^{1 / 6} \int_{\sigma(T)}^{T}\|\nabla \dot{u}(\cdot, t)\|_{L^{2}}^{2} d t \\
& \leq \frac{a}{2 \mu+\lambda}\left(t_{2}-t_{1}\right)+C(\bar{\rho}) C_{0}^{2 / 3}
\end{aligned}
$$

provided $C_{0} \leq \varepsilon_{2}$. Therefore, one can choose $N_{1}$ and $N_{0}$ in (2.10) as:

$$
N_{1}=\frac{a}{2 \mu+\lambda}, \quad N_{0}=C(\bar{\rho}) C_{0}^{2 / 3} .
$$

Note that

$$
g(\zeta)=-\frac{a \zeta}{2 \mu+\lambda}\left(\zeta^{\gamma}-\tilde{\rho}^{\gamma}\right) \leq-N_{1}=-\frac{a}{2 \mu+\lambda}, \quad \text { for all } \quad \zeta \geq \tilde{\rho}+1 .
$$

So one can set $\bar{\zeta}=\tilde{\rho}+1$ in (2.11). Lemma 2.4 and (3.46) thus yield that

$$
\sup _{t \in[\sigma(T), T]}\|\rho\|_{L^{\infty}} \leq \max \left\{\frac{3 \bar{\rho}}{2}, \tilde{\rho}+1\right\}+N_{0} \leq \frac{3 \bar{\rho}}{2}+C(\bar{\rho}) C_{0}^{2 / 3} \leq \frac{7 \bar{\rho}}{4},
$$

provided

$$
C_{0} \leq \varepsilon \triangleq \min \left\{\varepsilon_{2}, \varepsilon_{3}, \varepsilon_{4}\right\}, \quad \text { for } \varepsilon_{4} \triangleq\left(\frac{\bar{\rho}}{4 C(\bar{\rho})}\right)^{3 / 2} .
$$

The combination of (3.46) with (3.47) completes the proof of Lemma 3.5.

From now on, we will always assume that the initial energy $C_{0}$ satisfies (3.48) and the positive constant $C$ may depend on

$$
T,\left\|\rho_{0}^{1 / 2} g\right\|_{L^{2}},\|\nabla g\|_{L^{2}},\left\|\nabla u_{0}\right\|_{H^{2}},\left\|\rho_{0}-\tilde{\rho}\right\|_{H^{3}},\left\|P\left(\rho_{0}\right)-P(\tilde{\rho})\right\|_{H^{3}},
$$

besides $\mu, \lambda, \tilde{\rho}, a, \gamma, \bar{\rho}, \beta$ and $M$, where $g$ is as in (1.9).

Next, we will derive important estimates on the spatial gradient of the smooth solution $(\rho, u)$.

Lemma 3.6 The following estimates hold

$$
\begin{gathered}
\sup _{0 \leq t \leq T} \int \rho|\dot{u}|^{2} d x+\int_{0}^{T} \int|\nabla \dot{u}|^{2} d x d t \leq C, \\
\sup _{0 \leq t \leq T}\left(\|\nabla \rho\|_{L^{2} \cap L^{6}}+\|\nabla u\|_{H^{1}}\right)+\int_{0}^{T}\|\nabla u\|_{L^{\infty}} d t \leq C .
\end{gathered}
$$


Proof. Taking $\theta=1$ in (3.32) together with (3.36) gives

$$
\sup _{t \in[0, T]}\|\nabla u\|_{L^{2}}^{2}+\int_{0}^{T} \int \rho|\dot{u}|^{2} d x d t \leq C .
$$

Taking $m=0$ in (3.19), one can deduce from Gagliardo-Nirenberg's inequality (2.2), (2.6), (3.51) and (3.19) that

$$
\begin{aligned}
& \left(\int \rho|\dot{u}|^{2} d x\right)_{t}+\mu \int|\nabla \dot{u}|^{2} d x+(\mu+\lambda) \int(\operatorname{div} \dot{u})^{2} d x \\
& \leq C\|\nabla u\|_{L^{4}}^{4}+C(\bar{\rho})\|\nabla u\|_{L^{2}}^{2} \\
& \leq C\|\nabla u\|_{L^{2}}\|\nabla u\|_{L^{6}}^{3}+C \\
& \leq C\left(\|F\|_{L^{6}}^{3}+\|\omega\|_{L^{6}}^{3}+\|P-P(\tilde{\rho})\|_{L^{6}}^{3}\right)+C \\
& \leq C\left(\|\nabla F\|_{L^{2}}^{3}+\|\nabla \omega\|_{L^{2}}^{3}\right)+C \\
& \leq C\|\rho \dot{u}\|_{L^{2}}^{3}+C \\
& \leq C\left\|\rho^{1 / 2} \dot{u}\right\|_{L^{2}}^{4}+C .
\end{aligned}
$$

Taking into account on the compatibility condition (1.9), we can define

$$
\sqrt{\rho} \dot{u}(x, t=0)=\sqrt{\rho_{0}} g .
$$

Then (3.49) follows from (3.51)-(3.53) and Gronwall's inequality.

Next, we prove (3.50) by using Lemma 2.5 as in [17. For $2 \leq p \leq 6,|\nabla \rho|^{p}$ satisfies

$$
\begin{aligned}
& \left(|\nabla \rho|^{p}\right)_{t}+\operatorname{div}\left(|\nabla \rho|^{p} u\right)+(p-1)|\nabla \rho|^{p} \operatorname{div} u \\
& +p|\nabla \rho|^{p-2}(\nabla \rho)^{t} \nabla u(\nabla \rho)+p \rho|\nabla \rho|^{p-2} \nabla \rho \cdot \nabla \operatorname{div} u=0 .
\end{aligned}
$$

Thus,

$$
\begin{aligned}
\partial_{t}\|\nabla \rho\|_{L^{p}} & \leq C\left(1+\|\nabla u\|_{L^{\infty}}\right)\|\nabla \rho\|_{L^{p}}+C\left\|\nabla^{2} u\right\|_{L^{p}} \\
& \leq C\left(1+\|\nabla u\|_{L^{\infty}}\right)\|\nabla \rho\|_{L^{p}}+C\|\rho \dot{u}\|_{L^{p}}
\end{aligned}
$$

due to

$$
\left\|\nabla^{2} u\right\|_{L^{p}} \leq C\left(\|\rho \dot{u}\|_{L^{p}}+\|\nabla P\|_{L^{p}}\right),
$$

which follows from the standard $L^{p}$-estimate for the following elliptic system:

$$
\mu \Delta u+(\mu+\lambda) \nabla \operatorname{div} u=\rho \dot{u}+\nabla P, \quad u \rightarrow 0 \quad \text { as }|x| \rightarrow \infty .
$$

It follows from Lemma 2.5 and (3.55) that

$$
\begin{aligned}
\|\nabla u\|_{L^{\infty}} \leq & C\left(\|\operatorname{div} u\|_{L^{\infty}}+\|\omega\|_{L^{\infty}}\right) \log \left(e+\left\|\nabla^{2} u\right\|_{L^{6}}\right)+C\|\nabla u\|_{L^{2}}+C \\
\leq & C\left(\|\operatorname{div} u\|_{L^{\infty}}+\|\omega\|_{L^{\infty}}\right) \log \left(e+\|\dot{u}\|_{L^{6}}+\|\nabla P\|_{L^{6}}\right)+C \\
\leq & C\left(\|\operatorname{div} u\|_{L^{\infty}}+\|\omega\|_{L^{\infty}}\right) \log \left(e+\|\nabla \dot{u}\|_{L^{2}}\right) \\
& +C\left(\|\operatorname{div} u\|_{L^{\infty}}+\|\omega\|_{L^{\infty}}\right) \log \left(e+\|\nabla \rho\|_{L^{6}}\right)+C .
\end{aligned}
$$

Set

$f(t) \triangleq e+\|\nabla \rho\|_{L^{6}}, \quad g(t) \triangleq 1+\left(\|\operatorname{div} u\|_{L^{\infty}}+\|\omega\|_{L^{\infty}}\right) \log \left(e+\|\nabla \dot{u}\|_{L^{2}}\right)+\|\nabla \dot{u}\|_{L^{2}}$.

Combining (3.57) with (3.54) and setting $p=6$ in (3.54), one gets

$$
f^{\prime}(t) \leq C g(t) f(t)+C g(t) f(t) \ln f(t)+C g(t),
$$


which yields

$$
(\ln f(t))^{\prime} \leq C g(t)+C g(t) \ln f(t),
$$

due to $f(t)>1$. Note that (2.5), Lemma 2.2, (2.6), (3.49), and Lemma 3.5 imply

$$
\begin{aligned}
\int_{0}^{T} g(t) d t & \leq C \int_{0}^{T}\left(\|\operatorname{div} u\|_{L^{\infty}}^{2}+\|\omega\|_{L^{\infty}}^{2}\right) d t+C \\
& \leq C \int_{0}^{T}\left(\|F\|_{L^{\infty}}^{2}+\|P-P(\tilde{\rho})\|_{L^{\infty}}^{2}+\|\omega\|_{L^{\infty}}^{2}\right) d t+C \\
& \leq C \int_{0}^{T}\left(\|F\|_{L^{2}}^{2}+\|\nabla F\|_{L^{6}}^{2}+\|\omega\|_{L^{2}}^{2}+\|\nabla \omega\|_{L^{6}}^{2}\right) d t+C \\
& \leq C \int_{0}^{T}\|\nabla \dot{u}\|_{L^{2}}^{2} d t+C \\
& \leq C
\end{aligned}
$$

which, together with (3.58) and Gronwall's inequality, shows that

$$
\sup _{0 \leq t \leq T} f(t) \leq C
$$

Consequently,

$$
\sup _{0 \leq t \leq T}\|\nabla \rho\|_{L^{6}} \leq C
$$

As a consequence of (3.57), (3.59) and (3.60), one obtains

$$
\int_{0}^{T}\|\nabla u\|_{L^{\infty}} d t \leq C
$$

Next, taking $p=2$ in (3.54), one gets by using (3.61), (3.51) and Gronwall's inequality that

$$
\sup _{0 \leq t \leq T}\|\nabla \rho\|_{L^{2}} \leq C
$$

which, together with (3.55), (3.49), (3.51), (3.60), and (3.61), gives (3.50). The proof of Lemma 3.6 is completed.

The following Lemmas 3.7 3.10 will deal with the higher order estimates of the solutions which are needed to guarantee the extension of local classical solution to be a global one. The proofs are similar to the ones in [20], and we sketch them here for completeness.

Lemma 3.7 The following estimates hold

$$
\begin{aligned}
& \sup _{0 \leq t \leq T} \int \rho\left|u_{t}\right|^{2} d x+\int_{0}^{T} \int\left|\nabla u_{t}\right|^{2} d x d t \leq C, \\
& \sup _{t \in[0, T]}\left(\|\rho-\tilde{\rho}\|_{H^{2}}+\|P(\rho)-P(\tilde{\rho})\|_{H^{2}}\right) \leq C .
\end{aligned}
$$


Proof. Estimate (3.62) follows directly from the following simple facts:

$$
\begin{aligned}
\int \rho\left|u_{t}\right|^{2} d x & \leq \int \rho|\dot{u}|^{2} d x+\int \rho|u \cdot \nabla u|^{2} d x \\
& \leq C+C\left\|\rho^{1 / 2} u\right\|_{L^{2}}\|u\|_{L^{6}}\|\nabla u\|_{L^{6}}^{2} \\
& \leq C,
\end{aligned}
$$

and

$$
\begin{aligned}
\left\|\nabla u_{t}\right\|_{L^{2}}^{2} & \leq\|\nabla \dot{u}\|_{L^{2}}^{2}+\|\nabla(u \cdot \nabla u)\|_{L^{2}}^{2} \\
& \leq\|\nabla \dot{u}\|_{L^{2}}^{2}+C\|u\|_{L^{\infty}}^{2}\left\|\nabla^{2} u\right\|_{L^{2}}^{2}+C\|\nabla u\|_{L^{4}}^{4} \\
& \leq\|\nabla \dot{u}\|_{L^{2}}^{2}+C,
\end{aligned}
$$

due to Lemma 3.6 .

Next, we prove (3.63). Note that $P$ satisfies

$$
P_{t}+u \cdot \nabla P+\gamma P \operatorname{div} u=0,
$$

which, together with (1.1) 1 and a simple computation, yields that

$$
\begin{aligned}
& \frac{d}{d t}\left(\left\|\nabla^{2} P\right\|_{L^{2}}^{2}+\left\|\nabla^{2} \rho\right\|_{L^{2}}^{2}\right) \\
& \quad \leq C\left(1+\|\nabla u\|_{L^{\infty}}\right)\left(\left\|\nabla^{2} P\right\|_{L^{2}}^{2}+\left\|\nabla^{2} \rho\right\|_{L^{2}}^{2}\right)+C\|F\|_{H^{2}}^{2}+C\|\omega\|_{H^{2}}^{2}+C,
\end{aligned}
$$

where we have used the following simple fact:

$$
\begin{aligned}
\|\nabla u\|_{H^{m}} & \leq C\left(\|\operatorname{div} u\|_{H^{m}}+\|\omega\|_{H^{m}}\right) \\
& \leq C\left(\|F\|_{H^{m}}+\|\omega\|_{H^{m}}+\|P-P(\tilde{\rho})\|_{H^{m}}\right), \quad \text { for } \quad m=1,2 .
\end{aligned}
$$

Noticing that $F$ and $\omega$ satisfy (2.4), we get by the standard $L^{2}$-estimate for elliptic system, (3.49) and (3.50) that

$$
\begin{aligned}
\|F\|_{H^{2}}+\|\omega\|_{H^{2}} & \leq C\left(\|F\|_{L^{2}}+\|\omega\|_{L^{2}}+\|\rho \dot{u}\|_{L^{2}}+\|\nabla(\rho \dot{u})\|_{L^{2}}\right) \\
& \leq C\left(1+\|\nabla \rho\|_{L^{3}}\|\dot{u}\|_{L^{6}}+\|\nabla \dot{u}\|_{L^{2}}\right) \\
& \leq C\left(1+\|\nabla \dot{u}\|_{L^{2}}\right),
\end{aligned}
$$

which, together with (3.65), Lemma 3.6, and Gronwall's inequality, gives directly

$$
\sup _{t \in[0, T]}\left(\left\|\nabla^{2} P\right\|_{L^{2}}+\left\|\nabla^{2} \rho\right\|_{L^{2}}\right) \leq C .
$$

Thus the proof of Lemma 3.7 is completed.

Lemma 3.8 The following estimates hold:

$$
\begin{gathered}
\sup _{0 \leq t \leq T}\left(\left\|\rho_{t}\right\|_{H^{1}}+\left\|P_{t}\right\|_{H^{1}}\right)+\int_{0}^{T}\left(\left\|\rho_{t t}\right\|_{L^{2}}^{2}+\left\|P_{t t}\right\|_{L^{2}}^{2}\right) d t \leq C, \\
\sup _{0 \leq t \leq T} \int\left|\nabla u_{t}\right|^{2} d x+\int_{0}^{T} \int \rho u_{t t}^{2} d x d t \leq C .
\end{gathered}
$$


Proof. We first prove (3.66). One deduces from (3.64) and (3.50) that

$$
\left\|P_{t}\right\|_{L^{2}} \leq C\|u\|_{L^{\infty}}\|\nabla P\|_{L^{2}}+C\|\nabla u\|_{L^{2}} \leq C .
$$

Differentiating (3.64) yields

$$
\nabla P_{t}+u \cdot \nabla \nabla P+\nabla u \cdot \nabla P+\gamma \nabla P \operatorname{div} u+\gamma P \nabla \operatorname{div} u=0 .
$$

Hence, by (3.50) and (3.63), one gets

$$
\left\|\nabla P_{t}\right\|_{L^{2}} \leq C\|u\|_{L^{\infty}}\left\|\nabla^{2} P\right\|_{L^{2}}+C\|\nabla u\|_{L^{3}}\|\nabla P\|_{L^{6}}+C\left\|\nabla^{2} u\right\|_{L^{2}} \leq C .
$$

The combination of (3.68) with (3.69) implies

$$
\sup _{0 \leq t \leq T}\left\|P_{t}\right\|_{H^{1}} \leq C .
$$

Note that $P_{t t}$ satisfies

$$
P_{t t}+\gamma P_{t} \operatorname{div} u+\gamma P \operatorname{div} u_{t}+u_{t} \cdot \nabla P+u \cdot \nabla P_{t}=0 .
$$

Thus, one gets from (3.71) (3.70) (3.50) and (3.62) that

$$
\begin{aligned}
& \int_{0}^{T}\left\|P_{t t}\right\|_{L^{2}}^{2} d t \\
& \leq C \int_{0}^{T}\left(\left\|P_{t}\right\|_{L^{6}}\|\nabla u\|_{L^{3}}+\left\|\nabla u_{t}\right\|_{L^{2}}+\left\|u_{t}\right\|_{L^{6}}\|\nabla P\|_{L^{3}}+\left\|\nabla P_{t}\right\|_{L^{2}}\right)^{2} d t \\
& \leq C .
\end{aligned}
$$

One can handle $\rho_{t}$ and $\rho_{t t}$ similarly. Thus (3.66) is proved.

Next, we prove (3.67). Differentiating (1.1) 2 with respect to $t$, then multiplying the resulting equation by $u_{t t}$, one gets after integration by parts that

$$
\begin{aligned}
\frac{1}{2} & \frac{d}{d t} \int\left(\mu\left|\nabla u_{t}\right|^{2}+(\lambda+\mu)\left(\operatorname{div} u_{t}\right)^{2}\right) d x+\int \rho u_{t t}^{2} d x \\
= & \frac{d}{d t}\left(-\frac{1}{2} \int \rho_{t}\left|u_{t}\right|^{2} d x-\int \rho_{t} u \cdot \nabla u \cdot u_{t} d x+\int P_{t} \operatorname{div} u_{t} d x\right) \\
& +\frac{1}{2} \int \rho_{t t}\left|u_{t}\right|^{2} d x+\int\left(\rho_{t} u \cdot \nabla u\right)_{t} \cdot u_{t} d x-\int \rho u_{t} \cdot \nabla u \cdot u_{t t} d x \\
& \quad-\int \rho u \cdot \nabla u_{t} \cdot u_{t t} d x-\int P_{t t} \operatorname{div} u_{t} d x \\
\triangleq & \frac{d}{d t} I_{0}+\sum_{i=1}^{5} I_{i} .
\end{aligned}
$$

It follows from (1.1) $1,3.50),(3.66)$ and $(3.62)$ that

$$
\begin{aligned}
\left|I_{0}\right| & =\left.\left|-\frac{1}{2} \int \rho_{t}\right| u_{t}\right|^{2} d x-\int \rho_{t} u \cdot \nabla u \cdot u_{t} d x+\int P_{t} \operatorname{div} u_{t} d x \mid \\
& \leq\left.\left|\int \operatorname{div}(\rho u)\right| u_{t}\right|^{2} d x \mid+C\left\|\rho_{t}\right\|_{L^{3}}\|u \cdot \nabla u\|_{L^{2}}\left\|u_{t}\right\|_{L^{6}}+C\left\|P_{t}\right\|_{L^{2}}\left\|\nabla u_{t}\right\|_{L^{2}} \\
& \leq C \int \rho\left|u\left\|u_{t}\right\| \nabla u_{t}\right| d x+C\left\|\nabla u_{t}\right\|_{L^{2}} \\
& \leq C\|u\|_{L^{6}}\left\|\rho^{1 / 2} u_{t}\right\|_{L^{2}}^{1 / 2}\left\|u_{t}\right\|_{L^{6}}^{1 / 2}\left\|\nabla u_{t}\right\|_{L^{2}}+C\left\|\nabla u_{t}\right\|_{L^{2}} \\
& \leq \delta\left\|\nabla u_{t}\right\|_{L^{2}}^{2}+C_{\delta},
\end{aligned}
$$




$$
\begin{aligned}
2\left|I_{1}\right| & =\left.\left|\int \rho_{t t}\right| u_{t}\right|^{2} d x \mid \\
& =\left|\int\left(\rho_{t} u+\rho u_{t}\right) \cdot \nabla\left(\left|u_{t}\right|^{2}\right) d x\right| \\
& \leq C\left(\left\|\rho_{t}\right\|_{L^{3}}\|u\|_{L^{\infty}}+\left\|\rho^{1 / 2} u_{t}\right\|_{L^{2}}^{1 / 2}\left\|u_{t}\right\|_{L^{6}}^{1 / 2}\right)\left\|u_{t}\right\|_{L^{6}}\left\|\nabla u_{t}\right\|_{L^{2}} \\
& \leq C\left\|\nabla u_{t}\right\|_{L^{2}}^{2}+C\left\|\nabla u_{t}\right\|_{L^{2}}^{5 / 2} \\
& \leq C\left\|\nabla u_{t}\right\|_{L^{2}}^{4}+C,
\end{aligned}
$$

and

$$
\begin{aligned}
\left|I_{2}\right|= & \left|\int\left(\rho_{t} u \cdot \nabla u\right)_{t} \cdot u_{t} d x\right| \\
= & \left|\int\left(\rho_{t t} u \cdot \nabla u \cdot u_{t}+\rho_{t} u_{t} \cdot \nabla u \cdot u_{t}+\rho_{t} u \cdot \nabla u_{t} \cdot u_{t}\right) d x\right| \\
\leq & \left\|\rho_{t t}\right\|_{L^{2}}\|u \cdot \nabla u\|_{L^{3}}\left\|u_{t}\right\|_{L^{6}}+\left\|\rho_{t}\right\|_{L^{2}}\left\|\left|u_{t}\right|^{2}\right\|_{L^{3}}\|\nabla u\|_{L^{6}} \\
& +\left\|\rho_{t}\right\|_{L^{3}}\|u\|_{L^{\infty}}\left\|\nabla u_{t}\right\|_{L^{2}}\left\|u_{t}\right\|_{L^{6}} \\
\leq & C\left\|\rho_{t t}\right\|_{L^{2}}^{2}+C\left\|\nabla u_{t}\right\|_{L^{2}}^{2} .
\end{aligned}
$$

Cauchy's inequality gives

$$
\begin{aligned}
\left|I_{3}\right|+\left|I_{4}\right| & =\left|\int \rho u_{t} \cdot \nabla u \cdot u_{t t} d x\right|+\left|\int \rho u \cdot \nabla u_{t} \cdot u_{t t} d x\right| \\
& \leq C\left\|\rho^{1 / 2} u_{t t}\right\|_{L^{2}}\left(\left\|u_{t}\right\|_{L^{6}}\|\nabla u\|_{L^{3}}+\|u\|_{L^{\infty}}\left\|\nabla u_{t}\right\|_{L^{2}}\right) \\
& \leq \delta\left\|\rho^{1 / 2} u_{t t}\right\|_{L^{2}}^{2}+C_{\delta}\left\|\nabla u_{t}\right\|_{L^{2}}^{2}
\end{aligned}
$$

and

$$
\begin{aligned}
\left|I_{5}\right| & =\left|\int P_{t t} \operatorname{div} u_{t} d x\right| \\
& \leq\left\|P_{t t}\right\|_{L^{2}}\left\|\operatorname{div} u_{t}\right\|_{L^{2}} \\
& \leq C\left\|P_{t t}\right\|_{L^{2}}^{2}+\left\|\nabla u_{t}\right\|_{L^{2}}^{2} .
\end{aligned}
$$

Due to the regularity of the local solution, (2.1),$t \nabla u_{t} \in C\left(\left[0, T_{*}\right] ; L^{2}\right)$. Thus

$$
\begin{aligned}
\left\|\nabla u_{t}\left(\cdot, T_{*} / 2\right)\right\|_{L^{2}} & \leq \frac{2}{T_{*}}\left\|t \nabla u_{t}\right\|_{L^{\infty}\left(0, T_{*} ; L^{2}\right)} \\
& \leq C,
\end{aligned}
$$

where $C$ may also depend on $\|\nabla g\|_{L^{2}}$.

Collecting all the estimates (3.73)-(3.78), one deduces from (3.72), (3.66), (3.62) and Gronwall's inequality that

$$
\sup _{T_{*} / 2 \leq t \leq T}\left\|\nabla u_{t}\right\|_{L^{2}}+\int_{T_{*} / 2}^{T} \int \rho\left|u_{t t}\right|^{2} d x d t \leq C .
$$

On the other hand, (2.1) gives

$$
\sup _{0 \leq t \leq T_{*} / 2}\left\|\nabla u_{t}\right\|_{L^{2}}+\int_{0}^{T_{*} / 2} \int \rho\left|u_{t t}\right|^{2} d x d t \leq C .
$$

The combination of (3.79) with (3.80) yields (3.67) immediately. This completes the proof of Lemma 3.8 . 
Lemma 3.9 It holds that

$$
\begin{gathered}
\sup _{t \in[0, T]}\left(\|\rho-\tilde{\rho}\|_{H^{3}}+\|P-P(\tilde{\rho})\|_{H^{3}}\right) \leq C, \\
\sup _{t \in[0, T]}\left(\left\|\nabla u_{t}\right\|_{L^{2}}+\|\nabla u\|_{H^{2}}\right)+\int_{0}^{T}\left(\|\nabla u\|_{H^{3}}^{2}+\left\|\nabla u_{t}\right\|_{H^{1}}^{2}\right) d t \leq C .
\end{gathered}
$$

Proof. It follows from (3.67) and (3.50) that

$$
\begin{aligned}
\|\nabla(\rho \dot{u})\|_{L^{2}} \leq & \|\nabla \rho\| u_{t}\left\|_{L^{2}}+\right\| \rho \nabla u_{t}\left\|_{L^{2}}+\right\| \nabla \rho\|u\| \nabla u \mid \|_{L^{2}} \\
& +\left\|\rho|\nabla u|^{2}\right\|_{L^{2}}+\left\|\rho \left|u\left\|\nabla^{2} u \mid\right\|_{L^{2}}\right.\right. \\
\leq & \|\nabla \rho\|_{L^{3}}\left\|u_{t}\right\|_{L^{6}}+C\left\|\nabla u_{t}\right\|_{L^{2}}+C\|\nabla \rho\|_{L^{3}}\|u\|_{L^{\infty}}\|\nabla u\|_{L^{6}} \\
& +C\|\nabla u\|_{L^{3}}\|\nabla u\|_{L^{6}}+C\|u\|_{L^{\infty}}\left\|\nabla^{2} u\right\|_{L^{2}} \\
\leq & C,
\end{aligned}
$$

which together with (3.49) gives

$$
\sup _{0 \leq t \leq T}\|\rho \dot{u}\|_{H^{1}} \leq C
$$

The standard $H^{1}$-estimate for elliptic system (3.56) gives

$$
\begin{aligned}
\left\|\nabla^{2} u\right\|_{H^{1}} & \leq C\|\mu \Delta u+(\mu+\lambda) \nabla \operatorname{div} u\|_{H^{1}} \\
& =C\|\rho \dot{u}+\nabla P\|_{H^{1}} \\
& \leq C\left(\|\rho \dot{u}\|_{H^{1}}+\|\nabla P\|_{H^{1}}\right) \\
& \leq C
\end{aligned}
$$

due to $(1.1)_{2},(3.83)$ and $(3.63)$. As a consequence of (3.50) and (3.84), one has

$$
\sup _{0 \leq t \leq T}\|\nabla u\|_{H^{2}} \leq C
$$

Therefore, the standard $L^{2}$-estimate for elliptic system, (3.50), and Lemma 3.8 yield that

$$
\begin{aligned}
\left\|\nabla^{2} u_{t}\right\|_{L^{2}} \leq & C\left\|\mu \Delta u_{t}+(\mu+\lambda) \nabla \operatorname{div} u_{t}\right\|_{L^{2}} \\
= & \left\|\rho u_{t t}+\rho_{t} u_{t}+\rho_{t} u \cdot \nabla u+\rho u_{t} \cdot \nabla u+\rho u \cdot \nabla u_{t}+\nabla P_{t}\right\|_{L^{2}} \\
\leq & C\left(\left\|\rho u_{t t}\right\|_{L^{2}}+\left\|\rho_{t}\right\|_{L^{3}}\left\|u_{t}\right\|_{L^{6}}+\left\|\rho_{t}\right\|_{L^{3}}\|u\|_{L^{\infty}}\|\nabla u\|_{L^{6}}\right) \\
& +C\left(\left\|u_{t}\right\|_{L^{6}}\|\nabla u\|_{L^{3}}+\|u\|_{L^{\infty}}\left\|\nabla u_{t}\right\|_{L^{2}}+\left\|\nabla P_{t}\right\|_{L^{2}}\right) \\
\leq & C\left\|\rho u_{t t}\right\|_{L^{2}}+C,
\end{aligned}
$$

which, together with (3.67), implies

$$
\int_{0}^{T}\left\|\nabla u_{t}\right\|_{H^{1}}^{2} d t \leq C
$$

Applying the standard $H^{2}$-estimate for elliptic system (3.56) again leads to

$$
\begin{aligned}
\left\|\nabla^{2} u\right\|_{H^{2}} & \leq C\|\mu \Delta u+(\mu+\lambda) \nabla \operatorname{div} u\|_{H^{2}} \\
& \leq C\|\rho \dot{u}\|_{H^{2}}+C\|\nabla P\|_{H^{2}} \\
& \leq C+C\left\|\nabla u_{t}\right\|_{H^{1}}+C\left\|\nabla^{3} P\right\|_{L^{2}},
\end{aligned}
$$


where one has used (3.83) and the following simple facts:

$$
\begin{aligned}
\left\|\nabla^{2}\left(\rho u_{t}\right)\right\|_{L^{2}} & \leq C\left(\left\|\left|\nabla^{2} \rho\left\|u_{t}\left|\left\|_{L^{2}}+\right\|\right| \nabla \rho\right\| \nabla u_{t}\right|\right\|_{L^{2}}+\left\|\nabla^{2} u_{t}\right\|_{L^{2}}\right) \\
& \leq C\left(\left\|\nabla^{2} \rho\right\|_{L^{2}}\left\|\nabla u_{t}\right\|_{H^{1}}+\|\nabla \rho\|_{L^{3}}\left\|\nabla u_{t}\right\|_{L^{6}}+\left\|\nabla^{2} u_{t}\right\|_{L^{2}}\right) \\
& \leq C+C\left\|\nabla u_{t}\right\|_{H^{1}},
\end{aligned}
$$

and

$$
\begin{aligned}
\left\|\nabla^{2}(\rho u \cdot \nabla u)\right\|_{L^{2}} & \leq C\left(\left\|\left|\nabla^{2}(\rho u)\left\|\nabla u\left|\left\|_{L^{2}}+\right\|\right| \nabla(\rho u)\right\| \nabla^{2} u\right|\right\|_{L^{2}}+\left\|\nabla^{3} u\right\|_{L^{2}}\right) \\
& \leq C\left(1+\left\|\nabla^{2}(\rho u)\right\|_{L^{2}}\|\nabla u\|_{H^{2}}+\|\nabla(\rho u)\|_{L^{3}}\left\|\nabla^{2} u\right\|_{L^{6}}\right) \\
& \leq C\left(1+\left\|\nabla^{2} \rho\right\|_{L^{2}}\|u\|_{L^{\infty}}+\|\nabla \rho\|_{L^{6}}\|\nabla u\|_{L^{3}}+\left\|\nabla^{2} u\right\|_{L^{2}}\right) \\
& \leq C,
\end{aligned}
$$

due to (3.63) and (3.85). By using (3.85), (3.88), and (3.63), one may get that

$$
\begin{aligned}
& \left(\left\|\nabla^{3} P\right\|_{L^{2}}^{2}\right)_{t} \\
& \leq C\left(\left\|\left|\nabla^{3} u\left\|\nabla P\left|\left\|_{L^{2}}+\right\|\right| \nabla^{2} u\right\| \nabla^{2} P\right|\right\|_{L^{2}}+\left\|\left|\nabla u\left\|\nabla^{3} P \mid\right\|_{L^{2}}+\left\|\nabla^{4} u\right\|_{L^{2}}\right)\right\| \nabla^{3} P \|_{L^{2}}\right. \\
& \leq C\left(\left\|\nabla^{3} u\right\|_{L^{2}}\|\nabla P\|_{H^{2}}+\left\|\nabla^{2} u\right\|_{L^{3}}\left\|\nabla^{2} P\right\|_{L^{6}}+\|\nabla u\|_{L^{\infty}}\left\|\nabla^{3} P\right\|_{L^{2}}\right)\left\|\nabla^{3} P\right\|_{L^{2}} \\
& \quad+C\left(1+\left\|\nabla^{2} u_{t}\right\|_{L^{2}}+\left\|\nabla^{3} P\right\|_{L^{2}}\right)\left\|\nabla^{3} P\right\|_{L^{2}} \\
& \leq C+C\left\|\nabla u_{t}\right\|_{H^{1}}^{2}+C\left\|\nabla^{3} P\right\|_{L^{2}}^{2},
\end{aligned}
$$

which, together with Gronwall's inequality and (3.87), yields that

$$
\sup _{0 \leq t \leq T}\left\|\nabla^{3} P\right\|_{L^{2}} \leq C .
$$

Collecting all these estimates (3.87)-(3.89) and (3.63) shows

$$
\sup _{0 \leq t \leq T}\|P-P(\tilde{\rho})\|_{H^{3}}+\int_{0}^{T}\|\nabla u\|_{H^{3}}^{2} d t \leq C .
$$

It is easy to check similar arguments work for $\rho-\tilde{\rho}$ by using (3.90). Hence,

$$
\sup _{0 \leq t \leq T}\|\rho-\tilde{\rho}\|_{H^{3}} \leq C .
$$

Combing (3.90) with (3.91) shows (3.81). Estimate (3.82) thus follows from (3.67), (3.85), (3.87), and (3.90). Hence the proof of Lemma 3.9 is finished.

Lemma 3.10 For any $\tau \in(0, T)$, there exists some positive constant $C(\tau)$ such that

$$
\sup _{\tau \leq t \leq T}\left(\left\|\nabla u_{t}\right\|_{H^{1}}+\left\|\nabla^{4} u\right\|_{L^{2}}\right)+\int_{\tau}^{T} \int\left|\nabla u_{t t}\right|^{2} d x d t \leq C(\tau)
$$

Proof. Differentiate (1.1) 2 with respect to $t$ twice to get

$$
\begin{aligned}
& \rho u_{t t t}+\rho u \cdot \nabla u_{t t}-\mu \Delta u_{t t}-(\mu+\lambda) \nabla \operatorname{div} u_{t t} \\
& =2 \operatorname{div}(\rho u) u_{t t}+\operatorname{div}(\rho u)_{t} u_{t}-2(\rho u)_{t} \cdot \nabla u_{t}-\left(\rho_{t t} u+2 \rho_{t} u_{t}\right) \cdot \nabla u \\
& \quad-\rho u_{t t} \cdot \nabla u-\nabla P_{t t} .
\end{aligned}
$$


Multiplying (3.93) by $u_{t t}$ and then integrating the resulting equation over $\mathbb{R}^{3}$, one gets after integration by parts that

$$
\begin{aligned}
& \frac{1}{2} \frac{d}{d t} \int \rho\left|u_{t t}\right|^{2} d x+\int\left(\mu\left|\nabla u_{t t}\right|^{2}+(\mu+\lambda)\left(\operatorname{div} u_{t t}\right)^{2}\right) d x \\
& =-4 \int u_{t t}^{i} \rho u \cdot \nabla u_{t t}^{i} d x-\int(\rho u)_{t} \cdot\left[\nabla\left(u_{t} \cdot u_{t t}\right)+2 \nabla u_{t} \cdot u_{t t}\right] d x \\
& \quad-\int\left(\rho_{t t} u+2 \rho_{t} u_{t}\right) \cdot \nabla u \cdot u_{t t} d x-\int \rho u_{t t} \cdot \nabla u \cdot u_{t t} d x+\int P_{t t} \operatorname{div} u_{t t} d x \\
& \triangleq \sum_{i=1}^{5} J_{5} .
\end{aligned}
$$

We estimate each $J_{i}(i=1, \cdots, 5)$ as follows:

Hölder's inequality gives

$$
\begin{aligned}
\left|J_{1}\right| & \leq C\left\|\rho^{1 / 2} u_{t t}\right\|_{L^{2}}\left\|\nabla u_{t t}\right\|_{L^{2}}\|u\|_{L^{\infty}} \\
& \leq \delta\left\|\nabla u_{t t}\right\|_{L^{2}}^{2}+C_{\delta}\left\|\rho^{1 / 2} u_{t t}\right\|_{L^{2}}^{2} .
\end{aligned}
$$

It follows from (3.62), (3.66), (3.67), and (3.50) that

$$
\begin{aligned}
\left|J_{2}\right| & \leq C\left(\left\|\rho u_{t}\right\|_{L^{3}}+\left\|\rho_{t} u\right\|_{L^{3}}\right)\left(\left\|u_{t t}\right\|_{L^{6}}\left\|\nabla u_{t}\right\|_{L^{2}}+\left\|\nabla u_{t t}\right\|_{L^{2}}\left\|u_{t}\right\|_{L^{6}}\right) \\
& \leq C\left(\left\|\rho^{1 / 2} u_{t}\right\|_{L^{2}}^{1 / 2}\left\|u_{t}\right\|_{L^{6}}^{1 / 2}+\left\|\rho_{t}\right\|_{L^{6}}\|u\|_{L^{6}}\right)\left\|\nabla u_{t t}\right\|_{L^{2}} \\
& \leq \delta\left\|\nabla u_{t t}\right\|_{L^{2}}^{2}+C_{\delta}, \\
\left|J_{3}\right| & \leq C\left(\left\|\rho_{t t}\right\|_{L^{2}}\|u\|_{L^{\infty}}\|\nabla u\|_{L^{3}}+\left\|\rho_{t}\right\|_{L^{6}}\left\|u_{t}\right\|_{L^{6}}\|\nabla u\|_{L^{2}}\right)\left\|u_{t t}\right\|_{L^{6}} \\
& \leq \delta\left\|\nabla u_{t t}\right\|_{L^{2}}^{2}+C_{\delta}\left\|\rho_{t t}\right\|_{L^{2}}^{2},
\end{aligned}
$$

and

$$
\begin{aligned}
\left|J_{4}\right|+\left|J_{5}\right| & \leq C\left\|\rho u_{t t}\right\|_{L^{2}}\|\nabla u\|_{L^{3}}\left\|u_{t t}\right\|_{L^{6}}+C\left\|P_{t t}\right\|_{L^{2}}\left\|\nabla u_{t t}\right\|_{L^{2}} \\
& \leq \delta\left\|\nabla u_{t t}\right\|_{L^{2}}^{2}+C_{\delta}\left\|\rho^{1 / 2} u_{t t}\right\|_{L^{2}}^{2}+C_{\delta}\left\|P_{t t}\right\|_{L^{2}}^{2} .
\end{aligned}
$$

For any $\tau \in\left(0, T_{*}\right)$, since $t^{1 / 2} \sqrt{\rho} u_{t t} \in L^{\infty}\left(0, T_{*} ; L^{2}\right)$ by (2.1), there exists some $t_{0} \in$ $(\tau / 2, \tau)$ such that

$$
\begin{aligned}
\int \rho\left|u_{t t}\right|^{2} d x\left(t_{0}\right) & \leq \frac{1}{t_{0}}\left\|t^{1 / 2} \sqrt{\rho} u_{t t}\right\|_{L^{\infty}\left(0, T_{*} ; L^{2}\right)}^{2} \\
& \leq C(\tau) .
\end{aligned}
$$

Substituting (3.95)-(3.98) into (3.94) and choosing $\delta$ suitably small, one obtains by using (3.66) (3.99) and Gronwall's inequality that

$$
\sup _{t_{0} \leq t \leq T} \int \rho\left|u_{t t}\right|^{2} d x+\int_{t_{0}}^{T} \int\left|\nabla u_{t t}\right|^{2} d x d t \leq C(\tau),
$$

which, together with (3.86) and (3.67), yields that

$$
\sup _{\tau \leq t \leq T}\left\|\nabla u_{t}\right\|_{H^{1}}+\int_{\tau}^{T} \int\left|\nabla u_{t t}\right|^{2} d x d t \leq C(\tau)
$$

due to $t_{0}<\tau$. Now, (3.92) follows from (3.88), (3.100), and (3.81). We finish the proof of Lemma 3.10 . 


\section{Proof of Theorems 1.1 and 1.2}

With all the a priori estimates in Section 3 at hand, we are ready to prove the main results of this paper in this section.

Proof of Theorem 1.1. By Lemma 2.1, there exists a $T_{*}>0$ such that the Cauchy problem (1.1), (1.3), (1.4) has a unique classical solution $(\rho, u)$ on $\mathbb{R}^{3} \times\left(0, T_{*}\right]$. We will use the a priori estimates, Proposition 3.1 and Lemmas 3.9 and 3.10, to extend the local classical solution $(\rho, u)$ to all time.

First, it follows from (3.1), (3.2), (3.35) and (1.8) that

$$
A_{1}(0)+A_{2}(0)=0, \quad A_{3}(0) \leq C_{0}^{\delta_{0}}, \quad \rho_{0} \leq \bar{\rho},
$$

due to $C_{0} \leq \varepsilon$. Therefore, there exists a $T_{1} \in\left(0, T_{*}\right]$ such that (3.4) holds for $T=T_{1}$.

Next, we set

$$
T^{*}=\sup \{T \mid(\underline{3.4}) \text { holds }\} \text {. }
$$

Then $T^{*} \geq T_{1}>0$. Hence, for any $0<\tau<T \leq T^{*}$ with $T$ finite, it follows from Lemmas 3.9 and 3.10 that

$$
\nabla u_{t}, \nabla^{3} u \in C\left([\tau, T] ; L^{2} \cap L^{4}\right), \quad \nabla u, \nabla^{2} u \in C\left([\tau, T] ; L^{2} \cap C\left(\overline{\mathbb{R}^{3}}\right)\right),
$$

where we have used the standard embedding

$$
L^{\infty}\left(\tau, T ; H^{1}\right) \cap H^{1}\left(\tau, T ; H^{-1}\right) \hookrightarrow C\left([\tau, T] ; L^{q}\right), \quad \text { for any } q \in[2,6) .
$$

Due to (3.62), (3.67), and (3.92), one can get

$$
\begin{aligned}
& \int_{\tau}^{T}\left\|\left(\rho\left|u_{t}\right|^{2}\right)_{t}\right\|_{L^{1}} d t \\
& \leq \int_{\tau}^{T}\left(\left\|\rho_{t}\left|u_{t}\right|^{2}\right\|_{L^{1}}+2\left\|\rho u_{t} \cdot u_{t t}\right\|_{L^{1}}\right) d t \\
& \leq C \int_{\tau}^{T}\left(\left\|\rho\left|\operatorname{div} u\left\|\left.u_{t}\right|^{2}\right\|_{L^{1}}+\left\|\left|u\|\nabla \rho\| u_{t}\right|^{2}\right\|_{L^{1}}+\left\|\rho^{1 / 2} u_{t}\right\|_{L^{2}}\left\|\rho^{1 / 2} u_{t t}\right\|_{L^{2}}\right) d t\right.\right. \\
& \leq C \int_{\tau}^{T}\left(\left\|\rho\left|u_{t}\right|^{2}\right\|_{L^{1}}\|\nabla u\|_{L^{\infty}}+\|u\|_{L^{6}}\|\nabla \rho\|_{L^{2}}\left\|u_{t}\right\|_{L^{6}}^{2}+\left\|\rho^{1 / 2} u_{t t}\right\|_{L^{2}}\right) d t \\
& \leq C
\end{aligned}
$$

which yields

$$
\rho^{1 / 2} u_{t} \in C\left([\tau, T] ; L^{2}\right)
$$

This, together with (4.2), gives

$$
\rho^{1 / 2} \dot{u}, \nabla \dot{u} \in C\left([\tau, T] ; L^{2}\right) .
$$

Next, we claim that

$$
T^{*}=\infty .
$$

Otherwise, $T^{*}<\infty$. Then by Proposition 3.1. (3.5) holds for $T=T^{*}$. It follows from Lemmas 3.9 and 3.10 and (4.3) that $\left(\rho\left(x, T^{*}\right), u\left(x, T^{*}\right)\right)$ satisfies (1.7)-(1.9) except 
$u\left(\cdot, T^{*}\right) \in \dot{H}^{\beta}$, where $g(x) \triangleq \dot{u}\left(x, T^{*}\right), x \in \mathbb{R}^{3}$. Thus, Lemma 2.1 implies that there exists some $T^{* *}>T^{*}$, such that (3.4) holds for $T=T^{* *}$, which contradicts (4.1). Hence, (4.4) holds. Lemmas 2.1, 3.9 and 3.10 and (4.2) thus show that $(\rho, u)$ is in fact the unique classical solution defined on $\mathbb{R}^{3} \times(0, T]$ for any $0<T<T^{*}=\infty$.

Finally, to finish the proof of Theorem 1.1, it remains to prove (1.13).

Multiplying (3.40) by $4(P-P(\tilde{\rho}))^{3}$ and integrating the resulting equality over $\mathbb{R}^{3}$, one has

$$
\begin{aligned}
& \left(\|P-P(\tilde{\rho})\|_{L^{4}}^{4}\right)^{\prime}(t) \\
& =-(4 \gamma-1) \int(P-P(\tilde{\rho}))^{4} \operatorname{div} u d x-\gamma \int P(\tilde{\rho})(P-P(\tilde{\rho}))^{3} \operatorname{div} u d x,
\end{aligned}
$$

which yields that

$$
\int_{1}^{\infty}\left|\left(\|P-P(\tilde{\rho})\|_{L^{4}}^{4}\right)^{\prime}(t)\right| d t \leq C \int_{1}^{\infty}\left(\|P-P(\tilde{\rho})\|_{L^{4}}^{4}+\|\nabla u\|_{L^{4}}^{4}\right) d t \leq C,
$$

due to (3.43). Combining (3.43) with (4.5) leads to

$$
\lim _{t \rightarrow \infty}\|P-P(\tilde{\rho})\|_{L^{4}}=0,
$$

which together with (3.6) implies

$$
\lim _{t \rightarrow \infty} \int|\rho-\tilde{\rho}|^{q} d x=0
$$

for all $q$ satisfying (1.14). Note that (3.6) and (2.2) imply

$$
\int \rho^{1 / 2}|u|^{4} d x \leq\left(\int \rho|u|^{2} d x\right)^{1 / 2}\|u\|_{L^{6}}^{3} \leq C\|\nabla u\|_{L^{2}}^{3} .
$$

Thus (1.13) follows provided that

$$
\lim _{t \rightarrow \infty}\|\nabla u\|_{L^{2}}=0
$$

Setting

$$
I(t) \triangleq \frac{\mu}{2}\|\nabla u\|_{L^{2}}^{2}+\frac{\lambda+\mu}{2}\|\operatorname{div} u\|_{L^{2}}^{2},
$$

choosing $m=0$ in (3.9), and using (3.11) and (3.12), one has

$$
\left|I^{\prime}(t)\right| \leq C \int \rho|\dot{u}|^{2} d x+C\|\nabla u\|_{L^{3}}^{3}+C C_{0}^{1 / 2}\|\nabla \dot{u}\|_{L^{2}},
$$

where one has used the following simple estimate:

$$
\begin{aligned}
\left|M_{1}\right| & =\left|\int \dot{u} \cdot \nabla P d x\right| \\
& =\left|\int(P-P(\tilde{\rho})) \operatorname{div} \dot{u} d x\right| \\
& \leq C C_{0}^{1 / 2}\|\nabla \dot{u}\|_{L^{2}} .
\end{aligned}
$$


We thus deduce from (4.7), (3.36), and (3.43) that

$$
\begin{aligned}
\int_{1}^{\infty}\left|I^{\prime}(t)\right|^{2} d t & \leq C \int_{1}^{\infty}\left(\left\|\rho^{1 / 2} \dot{u}\right\|_{L^{2}}^{4}+\|\nabla u\|_{L^{2}}^{2}\|\nabla u\|_{L^{4}}^{4}+\|\nabla \dot{u}\|_{L^{2}}^{2}\right) d t \\
& \leq C \int_{1}^{\infty}\left(\left\|\rho^{1 / 2} \dot{u}\right\|_{L^{2}}^{2}+\|\nabla u\|_{L^{4}}^{4}+\|\nabla \dot{u}\|_{L^{2}}^{2}\right) d t \\
& \leq C
\end{aligned}
$$

which, together with

$$
\int_{1}^{\infty}|I(t)|^{2} d t \leq C \int_{1}^{\infty}\|\nabla u\|_{L^{2}}^{2} d t \leq C
$$

implies (4.6). The proof of Theorem 1.1 is finished.

Proof of Theorem 1.2. The proof is similar to that of Theorem 1.2 in [25]. We just sketch it here.

Otherwise, there exist some constant $C_{1}>0$ and a subsequence $\left\{t_{n_{j}}\right\}_{j=1}^{\infty}, t_{n_{j}} \rightarrow$ $\infty$ such that $\left\|\nabla \rho\left(\cdot, t_{n_{j}}\right)\right\|_{L^{r}} \leq C_{1}$. Hence, the Gagliardo-Nirenberg inequality (2.3) yields that there exists some positive constant $C$ independent of $t_{n_{j}}$ such that for $a=r /(2 r-3) \in(0,1)$,

$$
\begin{aligned}
& \left\|\rho\left(x, t_{n_{j}}\right)-\tilde{\rho}\right\|_{C\left(\overline{\mathbb{R}^{3}}\right)} \\
& \quad \leq C\left\|\nabla \rho\left(x, t_{n_{j}}\right)\right\|_{L^{r}}^{a}\left\|\rho\left(x, t_{n_{j}}\right)-\tilde{\rho}\right\|_{L^{3}}^{1-a} \\
& \quad \leq C C_{1}^{a}\left\|\rho\left(x, t_{n_{j}}\right)-\tilde{\rho}\right\|_{L^{3}}^{1-a} .
\end{aligned}
$$

Due to (1.13), the right hand side of (4.8) goes to 0 as $t_{n_{j}} \rightarrow \infty$. Hence,

$$
\left\|\rho\left(x, t_{n_{j}}\right)-\tilde{\rho}\right\|_{C\left(\overline{\mathbb{R}^{3}}\right)} \rightarrow 0 \text { as } t_{n_{j}} \rightarrow \infty
$$

On the other hand, since $(\rho, u)$ is a classical solution satisfying (1.12), there exists a unique particle path $x_{0}(t)$ with $x_{0}(0)=x_{0}$ such that

$$
\rho\left(x_{0}(t), t\right) \equiv 0 \text { for all } t \geq 0 .
$$

So, we conclude from this identity that

$$
\left\|\rho\left(x, t_{n_{j}}\right)-\tilde{\rho}\right\|_{C\left(\overline{\mathbb{R}^{3}}\right)} \geq\left|\rho\left(x_{0}\left(t_{n_{j}}\right), t_{n_{j}}\right)-\tilde{\rho}\right| \equiv \tilde{\rho}>0
$$

which contradicts (4.9). This completes the proof of Theorem 1.2.

\section{References}

[1] Beal, J. T., Kato, T., Majda. A.: Remarks on the breakdown of smooth solutions for the 3-D Euler equations. Commun. Math. Phys 94, 61-66 (1984)

[2] Bergh, J., Lofstrom, J.: Interpolation spaces, An introduction, Berlin-HeidelbergNew York:Springer-Verlag, (1976)

[3] Cho, Y., Choe, H. J., Kim, H.: Unique solvability of the initial boundary value problems for compressible viscous fluid. J. Math. Pures Appl. 83, 243-275 (2004) 
[4] Cho, Y., Jin, B.J.: Blow-up of viscous heat-conducting compressible flows, J. Math. Anal. Appl. 320(2), 819-826 (2006)

[5] Cho, Y., Kim, H.: On classical solutions of the compressible Navier-Stokes equations with nonnegative initial densities. Manuscript Math. 120, 91-129 (2006)

[6] Choe, H. J., Kim, H.: Strong solutions of the Navier-Stokes equations for isentropic compressible fluids. J. Differ. Eqs. 190, 504-523 (2003)

[7] Feireisl, E., Novotny, A., Petzeltová, H.: On the existence of globally defined weak solutions to the Navier-Stokes equations. J. Math. Fluid Mech. 3(4), 358-392 (2001)

[8] Fujita, H., Kato, T.: On the Navier-Stokes initial value problem I. Archiv Rat. Mech. Anal. 16, 269-315 (1964)

[9] Hoff, D.: Global existence for 1D, compressible, isentropic Navier-Stokes equations with large initial data. Trans. Amer. Math. Soc. 303(1), 169-181 (1987)

[10] Hoff, D: Global solutions of the Navier-Stokes equations for multidimensional compressible flow with discontinuous initial data. J. Differ. Eqs. 120(1), 215-254 (1995)

[11] Hoff, D.: Strong convergence to global solutions for multidimensional flows of compressible, viscous fluids with polytropic equations of state and discontinuous initial data. Arch. Rational Mech. Anal. 132, 1-14 (1995)

[12] Hoff, D.: Compressible flow in a half-space with Navier boundary conditions. J. Math. Fluid Mech. 7(3), 315-338 (2005)

[13] Hoff, D.: Dynamics of singularity surfaces for compressible, viscous flows in two space dimensions. Comm. Pure Appl. Math. 55(11), 1365-1407 (2002)

[14] Hoff, D., Santos, M. M.: Lagrangean structure and propagation of singularities in multidimensional compressible flow. Arch. Rational Mech. Anal. 188(3), 509-543 (2008)

[15] Hoff, D., Tsyganov, E.: Time analyticity and backward uniqueness of weak solutions of the Navier-Stokes equations of multidimensional compressible flow. J. Differ. Eqs. 245(10) 3068-3094 (2008)

[16] Huang, X. D., Li, J., Xin Z. P.: Blowup criterion for viscous barotropic flows with vacuum states. Commun. Math. Phys., In press.

[17] Huang, X. D., Li, J., Xin Z. P.: Serrin type criterion for the three-dimensional compressible flows. Preprint

[18] Huang, X. D., Li, J., Luo, Z., Xin Z. P.: Global existence and blowup phenomena for smooth solutions to the two-dimensional compressible flows. Preprint

[19] Huang, X. D., Li, J., Xin Z. P.: Global well-posedness for classical solutions to the multi-dimensional isentropic compressible Navier-Stokes system with vacuum on bounded domains. In preparation, 2010.

[20] Huang, X. D., Xin, Z. P.: A blow-up criterion for classical solutions to the compressible Navier-Stokes equations, Sci. in China, 53(3), 671-686 (2010) 
[21] Kazhikhov, A. V., Shelukhin, V. V.: Unique global solution with respect to time of initial-boundary value problems for one-dimensional equations of a viscous gas. Prikl. Mat. Meh. 41, 282-291 (1977)

[22] Kato, T.: Strong $L^{p}$-solutions of the Navier-Stokes equation in $R^{m}$, with applications to weak solutions. Math. Z. 187(4), 471-480 (1984)

[23] Koch, H., Tataru, D.: Well-posedness for the Navier-Stokes equations. Adv. Math. 157(1), 22-35 (2001)

[24] Ladyzenskaja, O. A., Solonnikov, V. A., Ural'ceva, N. N.: Linear and quasilinear equations of parabolic type, American Mathematical Society, Providence, RI (1968)

[25] Li, J., Xin, Z.: Some uniform estimates and blowup behavior of global strong solutions to the Stokes approximation equations for two-dimensional compressible flows. J. Differ. Eqs. 221(2), 275-308 (2006).

[26] Lions, P. L.: Mathematical topics in fluid mechanics. Vol. 2. Compressible models. New York: Oxford University Press (1998)

[27] Matsumura, A., Nishida, T.: The initial value problem for the equations of motion of viscous and heat-conductive gases. J. Math. Kyoto Univ. 20(1), 67-104 (1980)

[28] Nash, J.: Le problème de Cauchy pour les équations différentielles d'un fluide général. Bull. Soc. Math. France. 90,487-497 (1962)

[29] Rozanova, O.: Blow up of smooth solutions to the compressible Navier-Stokes equations with the data highly decreasing at infinity, J. Differ. Eqs. 245, 17621774 (2008)

[30] Salvi,R., Straskraba, I.: Global existence for viscous compressible fluids and their behavior as $t \rightarrow \infty$. J. Fac. Sci. Univ. Tokyo Sect. IA. Math. 40, 17-51 (1993)

[31] Serre, D.: Solutions faibles globales des équations de Navier-Stokes pour un fluide compressible. C. R. Acad. Sci. Paris Sér. I Math. 303, 639-642 (1986)

[32] Serre, D.: Sur l'équation monodimensionnelle d'un fluide visqueux, compressible et conducteur de chaleur. C. R. Acad. Sci. Paris Sér. I Math. 303, 703-706 (1986)

[33] Serrin, J.: On the uniqueness of compressible fluid motion, Arch. Rational. Mech. Anal. 3, 271-288 (1959)

[34] Xin, Z. P.: Blowup of smooth solutions to the compressible Navier-Stokes equation with compact density. Comm. Pure Appl. Math. 51, 229-240 (1998)

[35] Zlotnik, A. A.: Uniform estimates and stabilization of symmetric solutions of a system of quasilinear equations. Diff. Equations, 36, 701-716(2000) 